\title{
EFFECT OF WATER STRESS AND FOLIAR FEEDING WITH BORON AND ZINC UNDER NPK FERTILIZER LEVELS ON GROWTH AND YIELD OF THE NEW PROMISING COTTON GENOTYPE (GIZA 86 X 10229)
}

Emara M.A.A.; Abdel-Aal, S.A. Amal and S.A.F. Hamoda

Department of Agronomy, Cotton Research Institute, Agricultural Research Center, Giza, Egypt

\section{ABSTRACT}

Two field experiments were carried out at Sakha Agricultural Research Station at Kafr El-Sheikh Governorate, Egypt, on new promising cotton genotype (Giza 86 X 10229) during 2013 and 2014 seasons to study the effect of water stress and foliar feeding with boron and zinc under NPK fertilizer levels on growth, earliness, yield, yield components and some fiber quality. Each experiment was laid out in a split split-plot design with four replications. The main plots involved three irrigation intervals (two weeks, three weeks and four weeks) throughout the growing season. The sub-plots were allocated to three NPK levels $\left(60 \mathrm{~kg} \mathrm{~N}+22.5 \mathrm{~kg} \mathrm{P}_{2} \mathrm{O}_{5}+24 \mathrm{~kg} \mathrm{~K} 2 \mathrm{O} / \mathrm{fed} ; 75 \mathrm{~kg} \mathrm{~N}+30 \mathrm{~kg} \mathrm{P}_{2} \mathrm{O}_{5}+36 \mathrm{~kg}\right.$ $\mathrm{K}_{2} \mathrm{O} / \mathrm{fed}$ and $90 \mathrm{~kg} \mathrm{~N}+37.5 \mathrm{~kg} \mathrm{P}_{2} \mathrm{O}_{5}+48 \mathrm{~kg} \mathrm{~K}_{2} \mathrm{O} /$ fed ) The sub sub-plots involved four foliar feeding treatments with boron and zinc(control (without foliar application), foliar application of Zn-EDTA, foliar application of B-EDTA. and foliar application of Zn-EDTA + B-EDTA). The timing of foliar applications were at the start and peak of flowering stages.

The most important results obtained could be summarized as follows:

1) The obtained results revealed that increasing irrigation intervals to four weeks significantly decreased days to first flower, plant height at harvest, no. of fruiting branches/plant, no. of open bolls/plant, boll weight and seed cotton yield/fed. in both seasons and days to first open boll in one season only. While, irrigation intervals did not exhibit significant effect on no. of monopodia/plant, first fruiting node, earliness \%, seed index, lint presenting and fiber properties under study.

2) The obtained results revealed that the high NPK fertilizer level ( $90 \mathrm{~kg} \mathrm{~N}+37.5$ $\mathrm{kg} \mathrm{P}_{2} \mathrm{O}_{5}+48 \mathrm{~kg} \mathrm{~K} \mathrm{O} /$ fed.) significantly increased first fruiting node, days to first flower and first open boll, plant height at harvest, no. of fruiting branches/plant, no. of open bolls/plant, boll weight and seed cotton yield/fed and significantly decreased earliness \% in both seasons and did not exhibit significant effect on no. of monopodia/plant, seed index, lint presenting and fiber properties under study in both seasons.

3) The obtained results revealed that foliar feeding with boron and zinc mixture significantly increased no. of fruiting branches/plant, no. of open bolls/plant, boll weight and seed cotton yield/fed. While, micronutrients treatments did not exhibit significant effect on plant height at harvest, no. of monopodia/plant, first fruiting node, days to first flower and first open boll, earliness \%, seed index, lint presenting and fiber properties under study in both seasons.

4) The interaction between irrigation intervals and NPK fertilizer levels significantly affected days to first flower, plant height at harvest, earliness\%, no. of open bolls/plant, boll weight and seed cotton yield/fed in both seasons and no. of fruiting branches/plant in one season only and did not exhibit significant effect on first fruiting node, days to first open boll, seed index, lint presenting and fiber properties under study in both seasons.

Fayoum J. Agric. Res. \& Dev., Vol. 29, No.1, January, 2015 
Emara M.A.A. et al.,

5) The interaction between NPK fertilizer levels and foliar feeding with some micronutrients treatments significantly affected no. of open bolls/plant and seed cotton yield/fed in one season and no. of fruiting branches/plant and earliness \% in both seasons and did not exhibit a significant effect on the other traits under study in both seasons.

6) The interaction between irrigation intervals, NPK fertilizer levels and foliar feeding with some micronutrients treatments had a significant effect on no. of fruiting branches/plant and no. of open bolls/plant in 2013 season only and boll weight and seed cotton yield/fed in both seasons. While, did not exhibit significant effect on the other traits under study in both seasons.

Generally, results obtained revealed that irrigation every two weeks in combination with the high NPK fertilizer level $\left(90 \mathrm{~kg} \mathrm{~N}+37.5 \mathrm{~kg} \mathrm{P}_{2} \mathrm{O}_{5}+48 \mathrm{~kg}\right.$ $\mathrm{K}_{2} \mathrm{O} /$ fed.) and foliar feeding with $\mathrm{Zn}$ and $\mathrm{B}$ mixture $(2 \mathrm{~g}$ from each element/L water) at the start and peak of flowering stages for obtaining high productivity of the new promising genotype cotton (Giza 86 x 10229) under this study.

KEY WORDS: Cotton, Irrigation intervals, NPK fertilizer, Foliar feeding, Micronutrient, Boron, Zinc, Growth, Yield, Earliness and Fiber quality.

\section{INTRODUCTION}

Crop growth and yield are controlled by environmental factors (light, $\mathrm{CO}_{2}$ and temperature) and agricultural practices (water, nutrients and etc.) interacting with the genetically determined physiological and biochemical systems of the plant. Agricultural production strategy must be based on optimizing plant function in relation to environment to give high productivity with long-term stability.

Water management is one of the factors affecting the plant growth and productivity of cotton. In Egypt, the forthcoming water shortage, though it is currently not well recognized by the agro public, is a true challenge facing agricultural development and crop production in particular. Irrigation water applied less or more than the optimum requirement of a crop adversely affects the yield. It is, therefore, imperative to determine suitable time or proper stage of crop in appropriate amounts for application of irrigation water. Water deficiency particularly during fruiting stage markedly restricts over all plant growth, fruit retention, seed cotton yield, yield components and fiber quality Baslious and Abdel Malak (1992), El-Shahawy and Abd EL-Malik (1999), El-Sayed (2005), Hamed (2007), Ahmed and Kassem (2008), Halepyati et al., (2012), Hamoda et al., (2013) and Hamoda et al., (2014).

Through cotton agronomy programs, many traits are usually assigned to determine the optimum NPK fertilization levels for new promising cotton genotypes and commercial varieties. In this respect, several studies were done to evaluate the response of cotton plants to different NPK levels, Tomar et al., (2000), El-Ganaini et al., (2005), Hamed (2007), Policepatil et al., (2009), Hamoda et al., (2014) found that the plant height, no. of fruiting branches/plant, no. of bolls/plant, boll weight, seed index, seed cotton yield/plant and /fed. increased with increasing rates of NPK applied.

Some soil conditions in Egypt are perceived as being likely to induce micronutrients deficiencies such as high $\mathrm{pH}$, low organic matter and high calcium carbonate, (Hamissa and Abdel-Salam, 1999). Although, required by plants in small amounts, micronutrients play many complex roles in plant growth, plant nutrition, development and production. Micronutrients are involved in regulating plant physiology

Fayoum J. Agric. Res. \& Dev., Vol. 29, No.1, January, 2015 
EFFECT OF WATER STRESS AND FOLIAR FEEDING WITH BORON. and in enhancing plant stress tolerance, (El-Fouly and Fawzi 1995, Dar 2004, El-Fouly 2006, Malakouti 2006 and Wazir et al., 2013).

Boron (B) has been universally recognized as the most important micronutrient for cotton production, and cotton plant requires boron in relatively large amounts as compared with other plants (Roberts $\boldsymbol{e t}$ al., 2000 and Niaz et al., 2002). Boron helps in the biosynthesis of cell walls, and thereby cell division and elongation, in the rapidly growing, conductive and storage tissues; and also aids in sugars and nutrients translocation, resulting in promoting growth of vegetative growing tissues and developing storage sinks (Blevins and Lukaszewski, 1998). Boron deficiency during flowering and fruiting significantly reduced boll retention, resulting in lower yields (Gupta, 1993). Rosolem and Costa (1999) and Zhao and Oosterhuis (2003) showed that B deficiency in cotton decreased leaf photosynthesis and carbohydrate transport from leaves to developing fruit, and depressed plant growth, no. of reproductive structures and dry matter resulting in increased fruit abscission. Several workers documented favourable responses of cotton growth, productivity and fiber quality to foliar application with boron Oosterhuis and Venter, (1976), Sun and Xu, (1986), Gupta, (1993), Heitholt, (1994), Dong, (1995), Carvalho et al., (1996), Howard et al., (1998), Saeed (2000), El-Shazly et al., (2005) and El-Gabiery, (2014).

Zinc is an element which directly affects cotton yield and quality because of its function in biological membrane stability, enzyme activation ability, protein metabolism, photosynthetic carbon metabolism (Rengel, 2007 and Sema et al., 2012). Tolerance to environmental stresses has a high requirements for $\mathrm{Zn}$ and $\mathrm{Zn}$-deficient plants are sensitive to stress conditions, Cakmak (2000). Alloway(2008) reported that cotton is sensitive to $\mathrm{Zn}$ deficiency compared to some other crops such as wheat, oat, or pea. Moreover, its deficiency cause reduction in dry matter production of many crop plants (Wang and Jin, 2005 and El-Fouly, 2006). In this concern, Suresh and Kumar (2005), Sawan et al., (2006 and 2007), El-Menshawi and El-Sayed (2007), Kassem et al, (2009), Ali et al., (2011), Lale and Emine (2011), Sema et al., (2012), Emara (2012) and El-Gabiery, (2014) documented favourable responses of cotton growth, productivity and fiber quality to foliar application with zinc

The main objective of this investigation was to study the effect of water stress, through prolonging the irrigation interval and foliar feeding with boron and zinc under NPK fertilizer levels on growth, earliness, yield and yield components and fiber quality of the new promising cotton genotype (Giza 86 X 10229) in Sakha Agricultural Research Station at Kafr El-Sheikh Governorate, Egypt.

\section{MATERIALS AND METHODS}

Two field experiments were carried out at Sakha Agricultural Research Station at Kafr El-Sheikh Governorate, Egypt, during 2013 and 2014 seasons to study the response of the new promising cotton genotype (Giza 86 X 10229) belonging to (Gossypium barbadense, L.) to water stress and foliar feeding with boron and zinc under NPK fertilizer levels. Characterized the new promising cotton genotype (Giza 86 X 10229) are showed in Table (1). Each experiment was laid out in a split split-plot design with four replications. The main plots involved three irrigation intervals namely; A- Two weeks. BThree weeks and $\mathbf{C}$ - Four weeks throughout the growing season. The sub-plots were allocated to three levels of NPK namely; $1-60 \mathrm{~kg} \mathrm{~N}+22.5 \mathrm{~kg} \mathrm{P}_{2} \mathrm{O}_{5}+24 \mathrm{~kg} \mathrm{~K} \mathrm{~K}_{2} \mathrm{O} / \mathrm{fed}$. 2- 75 $\mathrm{kg} \mathrm{N}+30 \mathrm{~kg} \mathrm{P}_{2} \mathrm{O}_{5}+36 \mathrm{~kg} \mathrm{~K} 2 \mathrm{O} / \mathrm{fed}$. and 3- $90 \mathrm{~kg} \mathrm{~N}+37.5 \mathrm{~kg} \mathrm{P}_{2} \mathrm{O}_{5}+48 \mathrm{~kg} \mathrm{~K}_{2} \mathrm{O} /$ fed. The sub sub-plots involved the four treatments of foliar application with Zn-EDTA (14\%) and BEDTA (14\%) either alone or in mixtures which contain two elements at one level for each

Fayoum J. Agric. Res. \& Dev., Vol. 29, No.1, January, 2015 
Emara M.A.A. et al.,

$2 \mathrm{~g} / \mathrm{L}$ water. These treatments were; a- Control (without foliar application). b- Foliar application of $\mathrm{Zn}$. c- Foliar application of B. and d- Foliar application of $\mathrm{Zn}+\mathrm{B}$. The timing of foliar applications were at the start and peak of flowering stages.

Table (1): Characterized the cotton genotype (Giza 86 x 10229)

\begin{tabular}{|l|l|}
\hline Genotype name & New promising line (Giza 86 x 10229) \\
\hline Species & Barbadense. \\
\hline Category & Long staple and extra fine. \\
\hline Pedigree & Crossing between G86 x 10229. \\
\hline Characteristics & $\begin{array}{l}\text { Long staple characterized by high yielding, early maturity, resistance to } \\
\text { Fuzariam and high lint (\%). }\end{array}$ \\
\hline $\begin{array}{l}\text { Botanical } \\
\text { distinguishing } \\
\text { characters }\end{array}$ & $\begin{array}{l}\text { The stem has a medium length with polygon shape also has green color mixed } \\
\text { by dim red with medium length internodes. The leaves have palmate shape with } \\
\text { large size with no deep lobes and leather fell. The node of the first fruiting } \\
\text { branch ranged from 8 - 9. A flower petal has tubular shape. The boll size is } \\
\text { large and pyramid shape with drawn summit. Seed is big-sized and the fuzz } \\
\text { covers about fuzz less to } 1 / 4 \text { from the whole size and fuzz color is gray-greenish }\end{array}$ \\
\hline Hybrid bred by & Breeding Res. Section, Cotton Res. Inst., Agric. Res. Center, Giza, Egypt. \\
\hline
\end{tabular}

The sub sub-plot size was $18 \mathrm{~m}^{2}$ including 6 rows $(5 \mathrm{~m}$ long and $60 \mathrm{~cm}$ width). The distance between hills was $25 \mathrm{~cm}$. Cotton seeds were sown after two cuts of Egyptian clover Barseem (Trifolium alexandrinum L.,) in 2013 and 2014 seasons.

Soil samples were taken in the two seasons before planting cotton to estimate the soil characters using the standard methods as described by Chapman and Parker (1981). Mechanical, physical and chemical properties of the two experiment soil sites were presented in Table (1). In both seasons, the soil texture was clay loam. The results show that the two experiment soil sites had high $\mathrm{pH}$ and non-salinity. Organic matter and bicarbonate contents were low. Concerning soil macronutrients content, the soils of the two seasons were fairly low in total N, extractable-P, and low to medium in available K. Regarding soil micronutrients content, the soils of the two sites were high in available $\mathrm{Cu}$ but were poor in available contents of $\mathrm{Fe}, \mathrm{B}, \mathrm{Zn}$ and $\mathrm{Mn}$ measured by the critical levels according to Ankerman and Large (1974).

Table (2): Mechanical and chemical analysis of the experiment soil in 2013 and 2014 seasons.

\begin{tabular}{|c|c|c|c|c|c|c|c|c|c|c|c|c|c|}
\hline \multirow{3}{*}{ 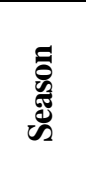 } & \multirow{3}{*}{ 苞 } & \multirow{3}{*}{ ㄴ. } & \multirow{3}{*}{ 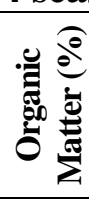 } & \multirow{3}{*}{$\begin{array}{l}8 \\
\mathscr{2} \\
0 \\
=\end{array}$} & \multirow{3}{*}{ बे } & \multicolumn{8}{|c|}{ Available elements (ppm) } \\
\hline & & & & & & \multicolumn{3}{|c|}{ Macro- Elements } & \multicolumn{5}{|c|}{ Micro-nutrients } \\
\hline & & & & & & $\mathbf{N}$ & $\mathbf{P}$ & $\mathbf{K}$ & $\mathbf{F e}$ & B & $\mathbf{Z n}$ & $\mathrm{Cu}$ & Mn \\
\hline 2013 & $\begin{array}{l}\text { Clay } \\
\text { loam }\end{array}$ & 7.70 & 1.69 & 0.64 & 1.82 & 12.10 & 9.21 & 131.2 & 4.3 & 1.21 & 1.66 & 2.77 & 3.1 \\
\hline 2014 & $\begin{array}{c}\text { Clay } \\
\text { loam }\end{array}$ & 8.38 & 1.74 & 0.69 & 1.81 & 11.95 & 9.50 & 126.2 & 5.2 & 1.16 & 1.18 & 2.62 & 2.8 \\
\hline
\end{tabular}

The first irrigation was applied after 21 day from planting irrigation, while the other irrigations were given at 14-days, 21-days and 28-days interval after the second irrigation. The other standard agricultural practices were followed throughout the two growing seasons.

Fayoum J. Agric. Res. \& Dev., Vol. 29, No.1, January, 2015 
Phosphorus in the form of superphosphate $\left(15.5 \% \quad \mathrm{P}_{2} \mathrm{O}_{5}\right)$ was applied during land preparation at the experimental treatments (rate of application). Nitrogen fertilizer in the form of ammonium nitrate $(33.5 \% \mathrm{~N})$ was added to submain plots according to the experimental treatments (rate of application) and divided into two equal doses i.e., the first one was applied after thinning just before the first irrigation and the second part before the second irrigation. Potassium in the form of potassium sulphate $\left(48 \% \mathrm{~K}_{2} \mathrm{O}\right)$ was added to sub-main plots according to the experimental treatments (rate of application).

In both seasons, five representative hills (10 plants/sub-main plot) were taken at random in order to study the following traits; plant height at harvest $(\mathrm{cm})$, no. of sympodia/plant, first sympodial position in nodes, days from sowing to the first flower, as well as to the first open boll, earliness percentage, no. of open bolls/plant, boll weight (g), seed cotton yield/plant (g), lint percentage and seed index $(\mathrm{g})$.

The yield of seed cotton in kentars/fed. was estimated from the three inner ridges, $($ One kentar $=157.5 \mathrm{~kg}$.). Fiber length parameters, micronaire reading and fiber strength were all determined individually. Fiber length parameters (Fiber upper half mean length (UHML), uniformity index (UI \%)) were determined on digital fibrograph instrument 630 according to A.S.T.M. D1447-07-2012. Micronaire reading was determined on micronaire instrument 675 according to A.S.T.M. D1448-97. Fiber strength was determined on Pressley instrument at zero gauge clamp spacing using a simple inclined plane breaker and simple specimen preparation and clamp loading techniques according to A.S.T.M.: D1445-1967. All fiber tests for the samples were made at the cotton laboratories under controlled atmospheric conditions according to ASTM (D 1776-04). Analysis of variance of the obtained data of each season was performed. The measured variables were analysed by ANOVA using M Stat-C statistical package (Freed, 1991). Mean comparisons were done using least significant differences (L.S.D) method at 5\% level $(\mathrm{P} \leq 0.05)$ of probability to compare differences between the means (Snedecor and Cochran, 1988).

\section{RESULTS AND DISCUSSION}

The results of growth traits, earliness parameters, yield and yield components as affected by water stress and foliar feeding with boron and zinc under NPK fertilizer levels and their interactions on new promising cotton genotype (Giza 86 X 10229) in Sakha Agricultural Research Station during 2013 and 2014 seasons are shown in Tables from (3) to (6).

\section{A- Growth traits:}

\section{A-1- Effect of irrigation intervals:}

Data in Table (3) showed that growth traits (plant height and no. of sympodia/plant) were significantly affected by irrigation intervals treatments. Irrigation every two weeks had significantly increased plant height (144.89 and $144.61 \mathrm{~cm})$ and no. of sympodia/plant (17.00 and 17.29) in 2013 and 2014 seasons, respectively compared with irrigation every three weeks or four weeks. While, no. of monopodia/plant was insignificantly affected by irrigation intervals

Fayoum J. Agric. Res. \& Dev., Vol. 29, No.1, January, 2015 
Emara M.A.A. et al.,

in both seasons. These results are in harmony with those obtained by El-Sayed (2005), Ahmed and Kassem (2008), Hamoda et al., (2014).

A-2- Effect of NPK levels :

Results presented in Table (3) indicate that levels of NPK had significant effect on growth traits (plant height and no. of sympodia/plant) and insignificant effect on no. of monopodia/plant in both seasons. The high level of NPK $(90 \mathrm{~kg} \mathrm{~N}$ $+37.5 \mathrm{~kg} \mathrm{P}_{2} \mathrm{O}_{5}+48 \mathrm{~kg} \mathrm{~K} 2 \mathrm{O} /$ fed.) significantly increased plant height (148.16 and $148.19 \mathrm{~cm}$ ) and no. of sympodia/plant (16.76 and 16.96) in 2013 and 2014 seasons, respectively, as compared with the other two rates. The positive response due to the high NPK rate on growth is mainly related to the followings :- N plays an important role in synthesis, distributing and accumulating the important substances responsible for growth and reflected greatly on dry weight plant. Such favourable effect of mineral $\mathrm{N}$ on dry matter accumulation might have been resulted from quickly provide the necessary $\mathrm{N}$ uptake in root zone, which resulted in more photosynthetic production and consequently increased dry matter accumulation (Hearn, 1981). In photosynthesis and respiration, $\mathrm{P}$ plays a major role in energy storage. Phosphorus works on organizing $\mathrm{pH}$ in plant cells because a large portion of it found as ions which works on keeping the hydrogen ion concentration at a level which makes the cell more active in (Uchida, 2000). Consequently, root system absorbs more nutrients in these favourable conditions which allow plants to grow better and more assimilates would be stored. These results are in harmony with those obtained by El-Ganaini et al., (2005), Policepatil et al., (2009) and Hamoda et al., (2014). In this concern, Seadh et al., (2012) found that plant height and number of fruiting branches were significantly increased by increasing NPK rate.

\section{A-3- Effect of micronutrients treatments:}

Results presented in Table (3) indicate that foliar application with micronutrients treatments had significant effect on no. of sympodia/plant and insignificant effect on plant height and no. of monopodia/plant in both seasons. The foliar feeding with boron and zinc mixture which contain two elements (Zn-EDTA and B-EDTA) at the start and peak of flowering stages significantly increased no. of sympodia/plant (16.63 and 16.86) in 2013 and 2014 seasons, respectively compared with the other micronutrients treatments. The constituents of nutrients mixture ( $\mathrm{Zn}$ and B) affect cotton plant growth, where Zinc is required in the synthesis of tryptophan, which, in turn, is necessary for the production of indole acetic acid in plants. Zinc is an essential component of several enzymes in plants variety dehydrogenases and, therefore, is necessary for several different functions in plant metabolism (Uchida, 2000). Boron is directly and indirectly involved in many physiological and biochemical processes during plant growth, such as cell elongation and division, cell wall biosynthesis, membrane function, nitrogen metabolism and photosynthesis (Blevins and Lukaszewski, 1998). These results are in harmony with those obtained by Saeed (2000), El-Shazly et al., (2005), Sawan et al., (2007), Kassem et al, (2009), Emara (2012) and El-Gabiery, (2014).

Fayoum J. Agric. Res. \& Dev., Vol. 29, No.1, January, 2015 
EFFECT OF WATER STRESS AND FOLIAR FEEDING WITH BORON.

Table (3): Cotton growth traits as affected by irrigation intervals, NPK levels and micronutrients treatments as well as their interactions during 2013 and 2014 seasons.

\begin{tabular}{|c|c|c|c|c|c|c|c|c|}
\hline \multicolumn{3}{|c|}{ Characters } & \multicolumn{2}{|c|}{$\begin{array}{c}\text { Plant height } \\
\text { at harvest }(\mathrm{cm})\end{array}$} & \multicolumn{2}{|c|}{$\begin{array}{c}\text { No. of } \\
\text { sympodia/plant }\end{array}$} & \multicolumn{2}{|c|}{$\begin{array}{c}\text { No. of } \\
\text { monopodia/plant }\end{array}$} \\
\hline \multicolumn{3}{|c|}{ Treatments } & \multirow[b]{2}{*}{2013} & \multirow[b]{2}{*}{2014} & \multirow[b]{2}{*}{2013} & \multirow[b]{2}{*}{2014} & \multirow[b]{2}{*}{2013} & \multirow[b]{2}{*}{2014} \\
\hline \begin{tabular}{|c|} 
Treatm \\
$\begin{array}{c}\text { Irrigation } \\
\text { intervals (A) }\end{array}$ \\
\end{tabular} & $\begin{array}{c}\text { Levels of NPK } \\
\text { (B) }\end{array}$ & Micronutrient (C) & & & & & & \\
\hline \multirow{15}{*}{ Two weeks } & \multirow{4}{*}{$\begin{array}{c}60 \mathrm{~N}+ \\
22.5 \mathrm{P}_{2} \mathrm{O}_{5}+24 \\
\mathrm{~K}_{2} \mathrm{O}\end{array}$} & Control & 141.00 & 139.33 & 16.30 & 16.56 & 1.50 & 1.50 \\
\hline & & Foliar Zn & 140.66 & 140.00 & 16.73 & 16.86 & 1.63 & 1.46 \\
\hline & & Foliar B & 139.66 & 140.66 & 16.83 & 16.83 & 1.50 & 1.43 \\
\hline & & Foliar Zn + B & 142.33 & 142.66 & 17.06 & 17.10 & 1.50 & 1.63 \\
\hline & \multicolumn{2}{|r|}{ Mean } & 140.91 & 140.66 & 16.73 & 16.84 & 1.53 & 1.48 \\
\hline & \multirow{4}{*}{$\begin{array}{c}75 \mathrm{~N}+ \\
30 \mathrm{P}_{2} \mathrm{O}_{5}+ \\
36 \mathrm{~K}_{2} \mathrm{O}\end{array}$} & Control & 144.00 & 144.00 & 17.40 & 17.20 & 1.46 & 1.50 \\
\hline & & Foliar Zn & 145.00 & 144.66 & 17.00 & 17.03 & 1.43 & 1.46 \\
\hline & & Foliar B & 144.66 & 143.66 & 16.90 & 17.23 & 1.33 & 1.50 \\
\hline & & Foliar Zn + B & 145.00 & 145.00 & 16.86 & 17.46 & 1.50 & 1.40 \\
\hline & \multicolumn{2}{|r|}{ Mean } & 144.66 & 144.33 & $\mathbf{1 7 . 0 4}$ & 17.23 & 1.43 & 1.46 \\
\hline & \multirow{4}{*}{$\begin{array}{c}90 \mathrm{~N}+ \\
37.5 \mathrm{P}_{2} \mathrm{O}_{5}+48 \\
\mathrm{~K}_{2} \mathrm{O}\end{array}$} & Control & 148.33 & 148.00 & 17.26 & 17.60 & 1.53 & 1.43 \\
\hline & & Foliar Zn & 149.00 & 149.00 & 16.93 & 17.73 & 1.40 & 1.46 \\
\hline & & Foliar B & 150.00 & 148.00 & 17.50 & 17.93 & 1.60 & 1.46 \\
\hline & & Foliar Zn + B & 149.00 & 149.00 & 17.23 & 17.93 & 1.66 & 1.46 \\
\hline & & 149.08 & 148.83 & 17.23 & 17.80 & 1.55 & $\mathbf{1 . 4 5}$ \\
\hline \multicolumn{3}{|c|}{ Mean two weeks } & 144.89 & 144.61 & 17.00 & 17.29 & 1.50 & 1.46 \\
\hline \multirow{15}{*}{ Three weeks } & \multirow{4}{*}{$\mid \begin{array}{c}60 \mathrm{~N}+ \\
22.5 \mathrm{P}_{2} \mathrm{O}_{5}+24 \\
\mathrm{~K}_{2} \mathrm{O}\end{array}$} & Control & 141.00 & 140.33 & 16.23 & 16.26 & 1.36 & 1.63 \\
\hline & & Foliar Zn & 141.33 & 140.33 & 16.40 & 16.63 & 1.56 & 1.40 \\
\hline & & Foliar B & 140.33 & 141.33 & 16.66 & 16.63 & 1.43 & 1.43 \\
\hline & & Foliar Zn + B & 139.66 & 141.00 & 16.63 & 16.70 & 1.36 & 1.60 \\
\hline & \multicolumn{2}{|c|}{ Mean } & 140.58 & 140.75 & 16.48 & 16.55 & 1.43 & 1.52 \\
\hline & \multirow{4}{*}{$\begin{array}{c}75 \mathrm{~N}+ \\
30 \mathrm{P}_{2} \mathrm{O}_{5}+36 \\
\mathrm{~K}_{2} \mathrm{O}\end{array}$} & Control & 144.66 & 144.66 & 16.70 & 16.70 & 1.46 & 1.53 \\
\hline & & Foliar Zn & 144.66 & 144.66 & 16.96 & 16.63 & 1.53 & 1.73 \\
\hline & & Foliar B & 144.00 & 144.66 & 17.00 & 16.70 & 1.53 & 1.43 \\
\hline & & Foliar Zn + B & 144.66 & 145.00 & 16.96 & 16.80 & 1.50 & 1.46 \\
\hline & \multicolumn{2}{|c|}{ Mean } & 144.50 & 144.58 & 16.90 & 16.70 & 1.50 & 1.54 \\
\hline & \multirow{4}{*}{$\begin{array}{c}90 \mathrm{~N}+ \\
37.5 \mathrm{P}_{2} \mathrm{O}_{5}+48 \\
\mathrm{~K}_{2} \mathrm{O}\end{array}$} & Control & 149.33 & 148.66 & 17.10 & 16.73 & 1.63 & 1.63 \\
\hline & & Foliar Zn & 149.00 & 149.66 & 17.10 & 16.90 & 1.66 & 1.46 \\
\hline & & Foliar B & 150.66 & 150.00 & 16.93 & 16.90 & 1.73 & 1.46 \\
\hline & & Foliar Zn + B & 147.66 & 148.66 & 17.23 & 17.16 & 1.63 & 1.43 \\
\hline & \multicolumn{2}{|c|}{ Mean } & 149.16 & 149.00 & 17.09 & 16.92 & 1.66 & 1.50 \\
\hline \multicolumn{3}{|c|}{ Mean three weeks } & 144.75 & 144.77 & 16.82 & 16.73 & 1.53 & 1.52 \\
\hline
\end{tabular}

Fayoum J. Agric. Res. \& Dev., Vol. 29, No.1, January, 2015 
Emara M.A.A. et al.,

Cont. Table (3):

\begin{tabular}{|c|c|c|c|c|c|c|c|c|}
\hline \multicolumn{3}{|c|}{ Characters } & \multicolumn{2}{|c|}{$\begin{array}{l}\text { Plant height at } \\
\text { harvest }(\mathrm{cm})\end{array}$} & \multicolumn{2}{|c|}{$\begin{array}{c}\text { No. of } \\
\text { sympodia/plant }\end{array}$} & \multicolumn{2}{|c|}{$\begin{array}{c}\text { No. of } \\
\text { monopodia/plant }\end{array}$} \\
\hline \multicolumn{3}{|c|}{ Seasons } & \multirow[b]{2}{*}{2013} & \multirow[b]{2}{*}{2014} & \multirow[b]{2}{*}{2013} & \multirow[b]{2}{*}{2014} & \multirow[b]{2}{*}{2013} & \multirow[b]{2}{*}{2014} \\
\hline $\begin{array}{c}\text { Treatments } \\
\begin{array}{c}\text { Irrigation } \\
\text { intervals (A) }\end{array} \\
\end{array}$ & $\begin{array}{l}\text { Levels of } \\
\text { NPK (B) }\end{array}$ & $\begin{array}{c}\text { Micronutrients } \\
\text { (C) }\end{array}$ & & & & & & \\
\hline \multirow{15}{*}{ Four weeks } & \multirow{4}{*}{$\mid \begin{array}{c}60 \mathrm{~N}+ \\
22.5 \mathrm{P}_{2} \mathrm{O}_{5}+ \\
24 \mathrm{~K}_{2} \mathrm{O}\end{array}$} & Control & 135.00 & 133.66 & 15.36 & 15.93 & 1.43 & 1.50 \\
\hline & & Foliar Zn & 133.66 & 133.33 & 15.53 & 16.20 & 1.53 & 1.46 \\
\hline & & Foliar B & 135.00 & 133.66 & 15.73 & 16.03 & 1.43 & 1.50 \\
\hline & & Foliar Zn + B & 136.33 & 133.33 & 15.86 & 16.16 & 1.46 & 1.46 \\
\hline & \multicolumn{2}{|r|}{ Mean } & 135.00 & 133.50 & 15.62 & 16.08 & 1.46 & 1.48 \\
\hline & \multirow{4}{*}{$\begin{array}{c}75 \mathrm{~N}+ \\
30 \mathrm{P}_{2} \mathrm{O}_{5}+ \\
36 \mathrm{~K}_{2} \mathrm{O}\end{array}$} & Control & 143.33 & 142.33 & 15.66 & 16.06 & 1.43 & 1.56 \\
\hline & & Foliar Zn & 144.00 & 144.00 & 15.60 & 15.93 & 1.60 & 1.46 \\
\hline & & Foliar B & 144.00 & 143.00 & 15.80 & 16.00 & 1.40 & 1.53 \\
\hline & & Foliar Zn + B & 143.66 & 144.00 & 15.90 & 16.20 & 1.50 & 1.33 \\
\hline & \multicolumn{2}{|r|}{ Mean } & 143.75 & 143.33 & 15.74 & 16.05 & 1.48 & 1.47 \\
\hline & \multirow{4}{*}{$\begin{array}{c}90 \mathrm{~N}+ \\
37.5 \mathrm{P}_{2} \mathrm{O}_{5} \\
48 \mathrm{~K}_{2} \mathrm{O}\end{array}$} & Control & 146.00 & 146.33 & 15.83 & 15.86 & 1.50 & 1.46 \\
\hline & & Foliar Zn & 147.00 & 147.00 & 16.03 & 16.40 & 1.56 & 1.46 \\
\hline & & Foliar B & 145.66 & 146.33 & 16.03 & 16.13 & 1.46 & 1.40 \\
\hline & & Foliar Zn + B & 146.33 & 147.33 & 15.93 & 16.26 & 1.46 & 1.40 \\
\hline & \multicolumn{2}{|r|}{ Mean } & 146.25 & 146.75 & 15.95 & 16.16 & 1.50 & 1.43 \\
\hline \multicolumn{3}{|c|}{ Mean four weeks } & 141.67 & 141.19 & 15.77 & 16.10 & 1.48 & 1.46 \\
\hline \multirow{3}{*}{$\begin{array}{l}\text { Mean levels of } \\
\text { NPK (B) }\end{array}$} & \multicolumn{2}{|c|}{$60 \mathrm{~N}+22.5 \mathrm{P}_{2} \mathrm{O}_{5}+24 \mathrm{~K}_{2} \mathrm{O}$} & 138.83 & 138.30 & 16.28 & 16.49 & 1.47 & 1.49 \\
\hline & \multicolumn{2}{|c|}{$75 \mathrm{~N}+30 \mathrm{P}_{2} \mathrm{O}_{5}+36 \mathrm{~K}_{2} \mathrm{O}$} & 144.30 & 144.08 & 16.56 & 16.66 & 1.48 & 1.49 \\
\hline & \multicolumn{2}{|c|}{$90 \mathrm{~N}+37.5 \mathrm{P}_{2} \mathrm{O}_{5}+48 \mathrm{~K}_{2} \mathrm{O}$} & 148.16 & 148.19 & 16.76 & 16.96 & 1.57 & 1.45 \\
\hline \multirow{4}{*}{$\begin{array}{c}\text { Mean } \\
\text { micronutrients } \\
\text { (C) }\end{array}$} & \multicolumn{2}{|c|}{$\begin{array}{c}\text { Control (without } \\
\text { micronutrients application) }\end{array}$} & 143.63 & 143.03 & 16.43 & 16.54 & 1.48 & 1.54 \\
\hline & \multicolumn{2}{|c|}{\begin{tabular}{|c|} 
Foliar $\mathbf{Z n}$ \\
\end{tabular}} & 143.81 & 143.63 & 16.47 & 16.70 & 1.54 & 1.48 \\
\hline & \multicolumn{2}{|c|}{ Foliar B } & 143.77 & 143.40 & 16.60 & 16.71 & 1.49 & 1.44 \\
\hline & \multicolumn{2}{|c|}{ Foliar Zn + B } & 143.85 & 144.03 & 16.63 & 16.86 & 1.51 & 1.46 \\
\hline & Irrigation & intervals (A) & 1.20 & 0.28 & $\mathbf{0 . 1 3}$ & 0.09 & N.S & N.S \\
\hline & Levels o & of NPK (B) & $\mathbf{0 . 8 3}$ & 0.78 & 0.10 & 0.07 & N.S & N.S \\
\hline & Micront & utrients $(C)$ & N.S & N.S & 0.11 & 0.13 & N.S & N.S \\
\hline LSD at 0.05 for & & $\mathbf{X ~ B}$ & 1.43 & 1.39 & N.S & 0.09 & N.S & N.S \\
\hline & & $\mathbf{X ~ C}$ & N.S & N.S & N.S & N.S & N.S & N.S \\
\hline & & $\mathbf{X ~ C}$ & N.S & N.S & 0.19 & 0.15 & N.S & N.S \\
\hline & $\mathbf{A X}$ & B X C & N.S & N.S & 0.34 & N.S & N.S & N.S \\
\hline
\end{tabular}

Fayoum J. Agric. Res. \& Dev., Vol. 29, No.1, January, 2015 
EFFECT OF WATER STRESS AND FOLIAR FEEDING WITH BORON 35 A-4- Effect of interaction:

Results presented in Table (3) indicate that interaction between irrigation intervals (A) and levels of NPK treatments (B) had significant effect on plant height in both seasons, no. of sympodia/plant in one season only and insignificant effect on no. of monopodia/plant in both seasons.

Data in Table (3) indicate that the interaction between irrigation intervals (A) and micronutrients treatments (C) gave insignificant effect on growth traits (plant height, no. of sympodia/plant and no. of monopodia/plant) in both seasons.

Results presented in Table (3) indicate that the interaction between levels of NPK treatments (B) and foliar application with micronutrients treatments (C) gave insignificant effect on no. of sympodia/plant and insignificant effect on plant height and no. of monopodia/plant in both seasons.

Data in Table (3) indicate that the interaction between (A) and (B) and (C) had significant effect on no. of sympodia/plant in one season only and insignificant effect on plant height and no. of monopodia/plant in both seasons.

\section{B- Earliness parameters:}

\section{B-1- Effect of irrigation intervals:}

The results in Table (4) show that, irrigation intervals treatments had a significant effect on earliness parameters; days to the first flower in both seasons and days to the first open boll in one season only, but gave insignificant effect on first sympodial position and earliness $\%$ in both seasons. Irrigation every two weeks significantly decreased days to the first flower (73.26 and 73.30 day) in 2013 and 2014 seasons, respectively, compared with irrigation every three weeks and four weeks. In this regard, El-Shahawy and Abd El-Malik (1999), El-Sayed (2005) and Hamoda et al., (2014) found that the earliness were insignificant affected by irrigation interval two weeks.

\section{B-2- Effect of levels of NPK:}

The results in Table (4) show that levels of NPK treatments had a significant effect on all earliness parameters (first sympodial position, days to the first flower and first open boll and earliness \%) in both seasons. The high level of NPK (90 kg N + $37.5 \mathrm{~kg} \mathrm{P}_{2} \mathrm{O}_{5}+48 \mathrm{~kg} \mathrm{~K} \mathrm{~K}_{2} \mathrm{O} /$ fed.) significantly increased, first sympodial position (5.46 and 5.41), days to the first flower (74.17 and 74.15 days), days to the first open boll (120.72 and 121.23 days). While significantly decreased earliness percentage (61.10 and 61.02\%) in 2013 and 2014 seasons, respectively, compared with the other levels. These results are in harmony with those obtained by El-Ganaini et al., (2005), Hamed (2007), Policepatil et al., (2009) and Hamoda et al., (2014).

\section{B-3- Effect of micronutrients treatments:}

Results presented in Table (4) indicate that foliar application with micronutrients treatments had insignificant effect on all earliness parameters; (first sympodial position, day to the first flower, days to the first open boll and earliness \%) in both seasons.

Fayoum J. Agric. Res. \& Dev., Vol. 29, No.1, January, 2015 
Emara M.A.A. et al.,

B-4- Effect of interaction:

Results presented in Table (4) indicate that the interaction between irrigation intervals (A) and levels of NPK (B) had significant effect on days to the first flower and earliness $\%$ and insignificant effect on (first sympodial position and days to the first open boll) in both seasons.

Table (4): Earliness parameters as affected by irrigation intervals, NPK levels and micronutrients treatments as well as their interactions during 2013 and 2014 seasons.

\begin{tabular}{|c|c|c|c|c|c|c|c|c|c|c|}
\hline \multicolumn{3}{|c|}{ Characters } & \multicolumn{2}{|c|}{$\begin{array}{c}\text { First sympodia } \\
\text { node }\end{array}$} & \multicolumn{4}{|c|}{$\begin{array}{l}\text { Pays to the firs pays to the firs } \\
\text { flower }\end{array}$} & \multicolumn{2}{|c|}{$\begin{array}{l}\text { Earliness } \\
\text { percentage }\end{array}$} \\
\hline \multirow{2}{*}{\multicolumn{3}{|c|}{ Seasons }} & \multirow[b]{3}{*}{2013} & \multirow[b]{3}{*}{2014} & \multirow[b]{3}{*}{2013} & \multirow[b]{3}{*}{2014} & \multirow[b]{3}{*}{2013} & \multirow[b]{3}{*}{2014} & \multirow[b]{3}{*}{2013} & \multirow[b]{3}{*}{2014} \\
\hline & & & & & & & & & & \\
\hline $\begin{array}{c}\text { Irrigation } \\
\text { intervals } \\
\text { (A) }\end{array}$ & Levels of NPK (B) & $\begin{array}{c}\text { Micronutrients } \\
\text { (C) }\end{array}$ & & & & & & & & \\
\hline \multirow{15}{*}{ Two weeks } & \multirow{4}{*}{$\begin{array}{c}60 \mathrm{~N}+ \\
22.5 \mathrm{P}_{2} \mathrm{O}_{5}+24 \mathrm{~K}_{2} \mathrm{O}\end{array}$} & Control & 5.30 & 5.23 & 72.63 & 72.76 & 119.70 & 119.93 & 63.50 & 63.66 \\
\hline & & Foliar Zn & 5.26 & 5.46 & 72.30 & 72.46 & 119.26 & 119.10 & 63.40 & 62.73 \\
\hline & & Foliar B & 5.40 & 5.50 & 72.53 & 72.53 & 120.26 & 119.36 & 63.16 & 63.53 \\
\hline & & Foliar Zn + B & 5.30 & 5.43 & 72.60 & 72.53 & 119.10 & 119.83 & 63.00 & 63.26 \\
\hline & \multicolumn{2}{|c|}{ Mean } & 5.31 & 5.40 & 72.51 & 72.57 & 119.58 & 119.55 & 63.26 & \begin{tabular}{|l|}
63.30 \\
\end{tabular} \\
\hline & \multirow{4}{*}{$\begin{array}{c}75 \mathrm{~N}+ \\
30 \mathrm{P}_{2} \mathrm{O}_{5}+ \\
36 \mathrm{~K}_{2} \mathrm{O}\end{array}$} & Control & 5.26 & 5.40 & \begin{tabular}{|l|}
73.16 \\
\end{tabular} & 73.46 & 119.33 & 119.73 & 61.90 & 62.43 \\
\hline & & Foliar Zn & 5.30 & 5.36 & \begin{tabular}{|l|}
73.13 \\
\end{tabular} & 73.43 & 119.80 & 120.10 & 61.63 & 61.60 \\
\hline & & Foliar B & 5.43 & 5.23 & \begin{tabular}{|l|}
72.93 \\
\end{tabular} & 72.90 & 119.83 & 120.70 & 61.93 & 62.16 \\
\hline & & Foliar Zn + B & 5.50 & 5.30 & \begin{tabular}{|l|l|}
73.16 \\
\end{tabular} & 72.93 & 119.63 & 119.43 & 62.43 & 62.06 \\
\hline & \multicolumn{2}{|c|}{ Mean } & 5.37 & 5.32 & \begin{tabular}{|l|}
73.10 \\
\end{tabular} & 73.18 & 119.65 & 119.99 & 61.97 & 62.06 \\
\hline & \multirow{4}{*}{$\begin{array}{c}90 \mathrm{~N}+ \\
37.5 \mathrm{P}_{2} \mathrm{O}_{5}+48 \mathrm{~K}_{2} \mathrm{O}\end{array}$} & Control & 5.40 & 5.43 & 74.23 & 74.20 & 120.86 & 121.66 & 61.23 & 60.73 \\
\hline & & Foliar Zn & 5.40 & 5.36 & 74.13 & 74.36 & 120.93 & 121.70 & 61.00 & 61.63 \\
\hline & & Foliar B & 5.43 & 5.40 & 74.06 & 74.10 & 120.73 & 121.23 & 61.53 & 61.40 \\
\hline & & Foliar Zn + B & 5.40 & 5.30 & 74.26 & 73.90 & 131.03 & 120.73 & 61.33 & 61.23 \\
\hline & \multicolumn{2}{|c|}{ Mean } & 5.40 & 5.40 & 74.17 & 74.14 & 120.89 & 121.33 & 61.27 & 61.25 \\
\hline \multicolumn{3}{|c|}{ Mean two weeks } & 5.36 & 5.38 & 73.26 & 73.30 & 120.04 & 120.29 & 62.17 & 62.20 \\
\hline \multirow{15}{*}{$\begin{array}{l}\text { Three } \\
\text { weeks }\end{array}$} & \multirow{4}{*}{$\begin{array}{c}60 \mathrm{~N}+ \\
22.5 \mathrm{P}_{2} \mathrm{O}_{5}+24 \mathrm{~K}_{2} \mathrm{O}\end{array}$} & Control & 5.36 & 5.43 & 72.63 & 72.66 & 119.63 & 120.40 & 63.23 & 63.03 \\
\hline & & Foliar Zn & 5.26 & 5.30 & 72.53 & 72.76 & 119.03 & 119.56 & 63.26 & 62.76 \\
\hline & & Foliar B & 5.30 & 5.30 & 72.83 & 72.66 & 119.73 & 120.43 & 63.20 & 63.40 \\
\hline & & Foliar Zn + B & 5.20 & 5.36 & 72.60 & 72.76 & 119.73 & 120.16 & 62.80 & 62.93 \\
\hline & \multicolumn{2}{|c|}{ Mean } & 5.28 & 5.35 & 72.65 & 72.71 & 119.53 & 120.14 & 63.12 & 63.03 \\
\hline & \multirow{4}{*}{$\begin{array}{c}75 \mathrm{~N}+ \\
30 \mathrm{P}_{2} \mathrm{O}_{5}+36 \mathrm{~K}_{2} \mathrm{O}\end{array}$} & Control & 5.36 & 5.33 & \begin{tabular}{|l|}
73.26 \\
\end{tabular} & 73.40 & 119.56 & 120.63 & 62.03 & 62.63 \\
\hline & & Foliar Zn & 5.30 & 5.26 & 73.23 & 73.63 & 119.30 & 120.50 & 62.36 & 62.50 \\
\hline & & Foliar B & 5.26 & 5.30 & \begin{tabular}{|l|}
73.10 \\
\end{tabular} & 73.13 & 119.70 & 120.10 & 62.33 & 62.53 \\
\hline & & Foliar Zn + B & 5.33 & 5.26 & \begin{tabular}{|l|}
73.50 \\
\end{tabular} & 73.10 & 120.50 & 120.63 & 62.63 & 62.13 \\
\hline & \multicolumn{2}{|c|}{ Mean } & 5.31 & 5.29 & \begin{tabular}{|l|}
73.27 \\
\end{tabular} & 73.31 & 119.76 & 120.46 & 62.34 & 62.45 \\
\hline & & Control & 5.43 & 5.43 & \begin{tabular}{|l|}
73.93 \\
\end{tabular} & 74.00 & 119.96 & 120.96 & 61.13 & 61.36 \\
\hline & & Foliar Zn & 5.53 & 5.46 & 73.83 & 73.86 & 120.50 & 121.30 & 61.23 & 60.90 \\
\hline & $37.5 \mathrm{P}_{2} \mathrm{O}_{5}+48 \mathrm{~K}_{2} \mathrm{O}$ & Foliar B & 5.36 & 5.36 & 73.90 & 73.80 & 120.60 & 121.30 & 61.06 & 61.06 \\
\hline & & Foliar Zn + B & 5.46 & 5.36 & \begin{tabular}{|l|}
74.03 \\
\end{tabular} & 73.80 & 120.26 & 120.60 & 61.13 & 61.03 \\
\hline & Mean & & 5.45 & 5.40 & 73.92 & 73.86 & 120.33 & 121.04 & 61.14 & 61.09 \\
\hline & Mean three week & & 5.35 & 5.35 & 73.28 & 73.30 & 119.87 & 120.55 & 62.20 & 62.19 \\
\hline
\end{tabular}

Fayoum J. Agric. Res. \& Dev., Vol. 29, No.1, January, 2015 
EFFECT OF WATER STRESS AND FOLIAR FEEDING WITH BORON.

Cont. Table (4):

\begin{tabular}{|c|c|c|c|c|c|c|c|c|c|c|}
\hline \multicolumn{3}{|c|}{ Characters } & \multicolumn{2}{|c|}{$\begin{array}{c}\text { First } \\
\text { sympodial } \\
\text { position }\end{array}$} & \multicolumn{2}{|c|}{$\begin{array}{l}\text { Days to the } \\
\text { first flower }\end{array}$} & \multicolumn{2}{|c|}{$\begin{array}{l}\text { pays to the firs } \\
\text { opened boll }\end{array}$} & \multicolumn{2}{|c|}{$\begin{array}{l}\text { Earliness } \\
\text { percentage }\end{array}$} \\
\hline \multicolumn{3}{|c|}{ Treatments } & \multirow[b]{2}{*}{2013} & \multirow[b]{2}{*}{2014} & \multirow[b]{2}{*}{2013} & \multirow[b]{2}{*}{2014} & \multirow[b]{2}{*}{2013} & \multirow[b]{2}{*}{2014} & \multirow[b]{2}{*}{2013} & \multirow[b]{2}{*}{2014} \\
\hline $\begin{array}{c}\text { Irrigation } \\
\text { intervals (A) }\end{array}$ & $\begin{array}{c}\text { Levels } \\
\text { of NPK } \\
\text { (B) } \\
\end{array}$ & $\begin{array}{c}\text { Micronutrients } \\
\text { (C) }\end{array}$ & & & & & & & & \\
\hline \multirow{15}{*}{ Four weeks } & \multirow{4}{*}{$\begin{array}{c}60 \mathrm{~N}+ \\
22.5 \\
\mathrm{P}_{2} \mathrm{O}_{5}+ \\
24 \mathrm{~K}_{2} \mathrm{O}\end{array}$} & Control & 5.26 & 5.23 & 72.60 & 72.53 & 120.63 & 119.56 & 63.33 & 63.26 \\
\hline & & Foliar Zn & 5.33 & 5.40 & 72.90 & 72.73 & 120.00 & 120.06 & 63.23 & 63.43 \\
\hline & & Foliar B & 5.26 & 5.30 & 72.70 & 72.76 & 119.66 & 120.23 & 63.16 & 63.10 \\
\hline & & Foliar Zn + B & 5.33 & 5.30 & 72.83 & 72.73 & 119.56 & 120.00 & 63.33 & 63.26 \\
\hline & \multicolumn{2}{|c|}{ Mean } & 5.30 & 5.30 & 72.75 & 72.69 & 119.96 & 119.96 & 63.26 & 63.26 \\
\hline & \multirow{4}{*}{$\begin{array}{c}75 \mathrm{~N}+ \\
30 \mathrm{P}_{2} \mathrm{O}_{5} \\
+ \\
36 \mathrm{~K}_{2} \mathrm{O}\end{array}$} & Control & 5.50 & 5.40 & 73.40 & 73.20 & 120.03 & 121.06 & 62.33 & 62.36 \\
\hline & & Foliar Zn & 5.23 & 5.33 & 73.16 & 73.13 & 119.46 & 120.43 & 62.20 & 62.40 \\
\hline & & Foliar B & 5.20 & 5.36 & 73.03 & 73.23 & 120.40 & 119.96 & 62.30 & 62.30 \\
\hline & & Foliar Zn + B & 5.23 & 5.16 & 73.40 & 73.16 & 120.60 & 120.63 & 62.16 & 62.26 \\
\hline & \multicolumn{2}{|c|}{ Mean } & 5.29 & 5.31 & 73.25 & 73.18 & 120.12 & 120.52 & 62.25 & 62.33 \\
\hline & \multirow{4}{*}{$\begin{array}{c}90 \mathrm{~N}+ \\
37.5 \\
\mathrm{P}_{2} \mathrm{O}_{5}+ \\
48 \mathrm{~K}_{2} \mathrm{O} \\
\end{array}$} & Control & 5.50 & 5.50 & 74.16 & 74.20 & 120.73 & 121.70 & 60.76 & 60.26 \\
\hline & & Foliar Zn & 5.60 & 5.46 & 74.56 & 74.60 & 120.80 & 121.50 & 60.86 & 61.03 \\
\hline & & Foliar B & 5.43 & 5.40 & 74.53 & 74.60 & 121.40 & 121.23 & 61.23 & 60.96 \\
\hline & & Foliar Zn + B & 5.60 & 5.40 & 74.46 & 74.40 & 120.83 & 120.93 & 60.73 & 60.66 \\
\hline & & Mean & 5.53 & 5.44 & 74.43 & $\mathbf{7 4 . 4 5}$ & 120.94 & $\mathbf{1 2 1 . 3 4}$ & 60.90 & 60.73 \\
\hline \multicolumn{3}{|c|}{ Mean four weeks } & 5.37 & 3.35 & $\mathbf{7 3 . 4 8}$ & 73.44 & 120.34 & 120.61 & 62.13 & 62.11 \\
\hline \multirow{3}{*}{$\begin{array}{c}\text { Mean levels } \\
\text { of NPK } \\
\text { (B) }\end{array}$} & \multicolumn{2}{|c|}{$60 \mathrm{~N}+22.5 \mathrm{P}_{2} \mathrm{O}_{5}+24 \mathrm{~K}_{2} \mathrm{O}$} & 5.30 & 5.35 & 72.64 & 72.66 & 119.69 & 119.88 & 63.21 & 63.20 \\
\hline & \multicolumn{2}{|c|}{$75 \mathrm{~N}+30 \mathrm{P}_{2} \mathrm{O}_{5}+36 \mathrm{~K}_{2} \mathrm{O}$} & 5.32 & 5.31 & 73.20 & 73.22 & 119.84 & 120.32 & 62.18 & 62.28 \\
\hline & \multicolumn{2}{|c|}{$90 \mathrm{~N}+37.5 \mathrm{P}_{2} \mathrm{O}_{5}+48 \mathrm{~K}_{2} \mathrm{O}$} & 5.46 & 5.41 & 74.17 & 74.15 & 120.72 & 121.23 & 61.10 & 61.02 \\
\hline \multirow{4}{*}{$\begin{array}{c}\text { Mean } \\
\text { micronutrien } \\
\text { (C) }\end{array}$} & \multicolumn{2}{|c|}{$\begin{array}{c}\text { Control (without } \\
\text { micronutrients application }\end{array}$} & 37 & 5.36 & 73.33 & 73.38 & 120.05 & 120.63 & 62.16 & 62.19 \\
\hline & & oliar Zn & 5.35 & 5.40 & 73.31 & 73.44 & 119.90 & 120.47 & 62.13 & 62.11 \\
\hline & & Foliar B & 5.34 & 5.34 & 73.29 & 73.30 & 120.25 & 120.50 & 62.21 & 62.27 \\
\hline & Fol & liar Zn + B & 5.37 & 5.33 & 73.43 & 73.25 & 120.14 & 120.33 & 62.17 & 62.09 \\
\hline & Irrigatic & on intervals (A) & N.S & N.S & 0.09 & 0.08 & 0.25 & N.S & N.S & N.S \\
\hline & Level & Is of NPK (B) & 0.06 & 0.07 & 0.10 & 0.11 & 0.25 & 0.30 & 0.15 & 0.16 \\
\hline & Micro & nutrients $(\mathrm{C})$ & N.S & N.S & N.S & N.S & N.S & N.S & N.S & N.S \\
\hline $\begin{array}{l}\text { LSD } \\
\text { for }\end{array}$ & & $\mathbf{A X ~ B ~}$ & N.S & N.S & 0.17 & 0.13 & N.S & N.S & N.S & 0.27 \\
\hline & & $\mathbf{A X C}$ & N.S & N.S & N.S & 0.23 & N.S & N.S & N.S & N.S \\
\hline & & B X C & N.S & N.S & N.S & N.S & N.S & N.S & 0.30 & 0.31 \\
\hline & & X B X C & N.S & N.S & N.S & N.S & N.S & N.S & N.S & N.S \\
\hline
\end{tabular}

Data in Table (4) indicate that the interaction between irrigation intervals (A) and foliar application with micronutrients treatments (C) gave insignificant effect on earliness parameters (first sympodial position, days to the first flower, days to the first open boll and earliness \%) in both seasons.

Results presented in Table (4) indicate that the interaction between levels of NPK treatments (B) and foliar application with micronutrients treatments (C) had significant effect on earliness $\%$ in both seasons. While, it had insignificant effect on (first sympodial position, days to the first flower and days to the first open boll) in both seasons.

Data in Table (4) indicate that the interaction between (A) and (B) and (C) gave insignificant effect on earliness parameters (first sympodial position, days to the first flower, days to the first open boll and earliness \%) in both seasons.

Fayoum J. Agric. Res. \& Dev., Vol. 29, No.1, January, 2015 
Emara M.A.A. et al.,

C- Yield and yield components:

C-1- Effect of irrigation intervals:

The results in Table (5) show that, irrigation intervals had a significant effect on no. of open bolls/plant, boll weight and seed cotton yield/feddan and insignificant effect on lint percentage and seed index in both seasons. The highest values of no. of bolls/plant (15.48 and 16.64), boll weight (3.10 and $3.11 \mathrm{~g}$ ) and seed cotton yield/feddan (11.64 and 12.51 kentar) were produced from irrigation every two weeks, while the lowest values of no. of bolls/plant (14.42 and 15.47), boll weight (2.95 and $2.96 \mathrm{~g}$ ) and seed cotton yield/feddan (10.28 and 11.06 kentar) were obtained from irrigation every four weeks, in 2013 and 2014 seasons, respectively. The increase in seed cotton yield due to this interaction is mainly due to the significance increase in plant height at harvest, no. of sympodia/plant, no. of open bolls/plant, boll weight and seed cotton yield/plant. These results are in accordance with those outlined by overall plant growth, fruit retention, seed cotton yield, yield components, El-Shahawy and Abd El-Malik (1999), ElSayed (2005), Ahmed and Kassem (2008) and Hamoda et al., (2013).

\section{C-2- Effect of NPK levels:}

The results in Table (5) show that, levels of NPK had a significant effect on no. of open bolls/plant, boll weight and seed cotton yield/feddan and insignificant effect on lint percentage and seed index in both seasons. The highest values of no. of bolls/plant (15.33 and 16.38), boll weight (3.08 and $3.08 \mathrm{~g}$ ) and seed cotton yield/feddan (11.45 and 12.23 kentar) were produced from the level of NPK ( $90 \mathrm{~kg} \mathrm{~N}+37.5 \mathrm{~kg} \mathrm{P}_{2} \mathrm{O}_{5}+48 \mathrm{~kg} \mathrm{~K} 2 \mathrm{O} /$ fed.), while the lowest values of no. of bolls/plant (14.59 and 15.84), boll weight (2.96 and $2.96 \mathrm{~g})$ and seed cotton yield/feddan (10.46 and 11.31 kentar) were obtained from the high level of NPK (60 kg $\mathrm{N}+22.5 \mathrm{~kg} \mathrm{P}_{2} \mathrm{O}_{5}+24 \mathrm{~kg} \mathrm{~K} \mathrm{O}_{2}$ /fed.), in 2013 and 2014 seasons, respectively. The positive response to the high NPK level with regard to seed cotton yield and its components might be due to the improvement nutrient availability and increases in nutrients uptake, the role of these two concentrations to increase leaf $\mathrm{N}, \mathrm{P}$ and $\mathrm{K}$ content and consequently increase photosynthesis, assimilates accumulation and plant dry weight and the higher number of open bolls/plant, heavier bolls and higher seed cotton yield per plant. The boll weight and seed index increases due to the high NPK level was mainly attributed to increase photosynthetic activity of cotton plants and consequently increase accumulation of metabolites with direct impact on boll weight and seed index. These results are in accordance with those outlined by overall plant growth, fruit retention, seed cotton yield, yield components, El-Ganaini et al., (2005) and Hamoda et al., (2014).

Fayoum J. Agric. Res. \& Dev., Vol. 29, No.1, January, 2015 
EFFECT OF WATER STRESS AND FOLIAR FEEDING WITH BORON. 39

Table (5): Cotton yield components as affected by irrigation intervals, NPK levels and micronutrients treatments as well as their interactions during 2013 and 2014 seasons.

\begin{tabular}{|c|c|c|c|c|c|c|c|c|c|c|c|c|}
\hline \multicolumn{3}{|c|}{ Characters } & \multicolumn{2}{|c|}{$\begin{array}{l}\text { No. of open } \\
\text { bolls/plant }\end{array}$} & \multicolumn{2}{|c|}{$\begin{array}{c}\text { Boll weight } \\
\text { (g) }\end{array}$} & \multicolumn{2}{|c|}{\begin{tabular}{|} 
Seed cotton \\
yield \\
Kentar/fed.
\end{tabular}} & \multicolumn{2}{|c|}{\begin{tabular}{|c|} 
Lint \\
percentage \\
$(\%)$ \\
\end{tabular}} & \multicolumn{2}{|c|}{$\begin{array}{c}\text { Seed index } \\
\text { (g) }\end{array}$} \\
\hline \multicolumn{3}{|c|}{ Treatments } & \multirow[b]{2}{*}{$\sim 2013$} & \multirow[b]{2}{*}{2014} & \multirow[b]{2}{*}{2013} & \multirow[b]{2}{*}{2014} & \multirow[b]{2}{*}{2013} & \multirow[b]{2}{*}{2013} & \multirow[b]{2}{*}{2014} & \multirow[b]{2}{*}{2013} & \multirow[b]{2}{*}{2014} & \multirow[b]{2}{*}{2013} \\
\hline $\begin{array}{c}\text { Irrigation } \\
\text { intervals } \\
\text { (A) } \\
\end{array}$ & $\begin{array}{c}\text { Levels } \\
\text { of NPK } \\
\text { (B) }\end{array}$ & $\begin{array}{l}\text { Micronutrie } \\
\text { nts (C) }\end{array}$ & & & & & & & & & & \\
\hline \multirow{15}{*}{$\begin{array}{c}\text { Two } \\
\text { weeks }\end{array}$} & \multirow{4}{*}{$\begin{array}{c}60 \mathrm{~N}+ \\
22.5 \\
\mathrm{P}_{2} \mathrm{O}_{5} \\
+24 \mathrm{~K}_{2} \mathrm{O}\end{array}$} & Control & 14.86 & 15.70 & 2.96 & 3.00 & 10.60 & 11.43 & 39.70 & 39.46 & 12.61 & 12.58 \\
\hline & & Foliar Zn & 14.93 & 15.93 & 2.98 & 3.01 & 10.75 & 11.63 & 39.46 & 39.96 & 12.62 & 12.72 \\
\hline & & Foliar B & 14.96 & 16.20 & 3.01 & 3.03 & 10.93 & 11.87 & 39.59 & 39.41 & 12.64 & 12.84 \\
\hline & & Foliar Zn + B & 14.96 & 16.53 & 3.07 & 3.04 & 11.16 & 12.11 & 39.43 & 39.36 & 12.65 & 12.59 \\
\hline & \multicolumn{2}{|c|}{ Mean } & 14.93 & 16.09 & 3.00 & 3.02 & 10.86 & 11.76 & 39.55 & 39.30 & 12.63 & 12.68 \\
\hline & \multirow{4}{*}{$\begin{array}{c}75 \mathrm{~N}+ \\
30 \mathrm{P}_{2} \mathrm{O}_{5} \\
+ \\
36 \mathrm{~K}_{2} \mathrm{O}\end{array}$} & Control & 15.06 & 16.46 & 3.08 & 3.10 & 11.34 & 12.22 & 39.41 & 39.49 & 12.72 & 12.78 \\
\hline & & Foliar Zn & 15.30 & 16.50 & 3.10 & 3.12 & 11.48 & 12.83 & 39.64 & 39.50 & 12.74 & 12.80 \\
\hline & & Foliar B & 15.53 & 16.60 & 3.11 & 3.11 & 11.71 & 12.58 & 39.39 & 39.55 & 12.75 & 12.76 \\
\hline & & Foliar Zn + B & 15.73 & 16.93 & 3.13 & 3.13 & 11.91 & 12.81 & 39.65 & 39.76 & 12.78 & 12.74 \\
\hline & \multicolumn{2}{|c|}{ Mean } & 15.40 & 16.62 & 3.10 & 3.11 & 11.61 & 12.50 & 39.52 & 39.57 & 12.75 & 12.77 \\
\hline & \multirow{4}{*}{$\begin{array}{c}90 \mathrm{~N}+ \\
37.5 \\
\mathrm{P}_{2} \mathrm{O}_{5}+ \\
48 \mathrm{~K}_{2} \mathrm{O}\end{array}$} & Control & 16.06 & 16.90 & 3.17 & 3.20 & 12.35 & 13.12 & 39.55 & 39.49 & 12.70 & 12.73 \\
\hline & & Foliar Zn & 16.13 & 17.16 & 3.18 & 3.22 & 12.39 & 13.19 & 39.35 & 39.67 & 12.68 & 12.59 \\
\hline & & Foliar B & 16.13 & 17.33 & 3.20 & 3.21 & 12.46 & 13.32 & 39.63 & 39.60 & 12.71 & 12.73 \\
\hline & & Foliar Zn + B & 16.16 & 17.43 & 3.22 & 3.22 & 12.63 & 13.51 & 39.53 & 39.63 & 12.70 & 12.62 \\
\hline & \multicolumn{2}{|c|}{ Mean } & 16.12 & 17.20 & 3.19 & 3.21 & 12.45 & 13.28 & 39.51 & 39.59 & 12.70 & 12.67 \\
\hline \multicolumn{3}{|c|}{ Mean two weeks } & 15.48 & 16.64 & 3.10 & 3.11 & 11.64 & $12 . .51$ & 39.53 & 39.49 & 12.69 & 12.70 \\
\hline & $60 \mathrm{~N}+$ & Control & 14.43 & 15.63 & 2.97 & 2.99 & 10.35 & 11.28 & 39.69 & 39.73 & 12.64 & 12.76 \\
\hline & 22.5 & Foliar Zn & 14.56 & 15.83 & 2.98 & 3.01 & 10.61 & 11.45 & 39.59 & 39.66 & 12.71 & 12.65 \\
\hline & $\mathbf{P}_{2} \mathbf{O}_{5}+$ & Foliar B & 14.73 & 15.90 & 3.00 & 3.00 & 10.64 & 11.51 & 39.56 & 39.46 & 12.62 & 12.61 \\
\hline & $24 \mathrm{~K}_{2} \mathrm{O}$ & Foliar Zn + B & 14.83 & 16.33 & 3.00 & 3.02 & 11.01 & 11.80 & 39.75 & 39.71 & 12.70 & 12.62 \\
\hline & & & 14.64 & 15.92 & 2.99 & 3.00 & 10.65 & 11.51 & 39.65 & 39.54 & 12.67 & 12.66 \\
\hline & $75 \mathrm{~N}+$ & Control & 14.90 & 16.10 & 3.02 & 3.04 & 11.05 & 11.86 & 39.44 & 39.31 & 12.64 & 12.67 \\
\hline & $\Omega$ & Foliar Zn & 15.00 & 15.93 & 3.04 & 3.06 & 11.03 & 11.83 & 39.59 & 39.25 & 12.74 & 12.76 \\
\hline $\begin{array}{l}\text { Three } \\
\text { weeks }\end{array}$ & $\mathbf{P}_{2} \mathbf{O}_{5}+$ & Foliar B & 15.03 & 16.13 & 3.05 & 3.06 & 11.06 & 11.91 & 39.53 & 39.76 & 12.72 & 12.74 \\
\hline & $36 \mathrm{~K}_{2} \mathrm{O}$ & Foliar Zn + B & 15.10 & 16.20 & 3.06 & 3.07 & 11.17 & 11.97 & 39.54 & 39.66 & 12.73 & 12.75 \\
\hline & & ean & 15.00 & 16.09 & 3.04 & 3.06 & 11.08 & 11.89 & $\mathbf{3 9 . 5 3}$ & 39.49 & 12.70 & 12.73 \\
\hline & $90 \mathrm{~N}+$ & Control & 15.03 & 16.20 & 3.01 & 3.01 & 11.01 & 11.82 & 39.61 & 39.48 & 12.68 & 12.76 \\
\hline & 37.5 & Foliar Zn & 15.13 & 16.40 & 3.04 & 3.04 & 11.15 & 11.95 & 39.49 & 39.72 & 12.65 & 12.59 \\
\hline & $\mathbf{P}_{2} \mathbf{O}_{5}+$ & Foliar B & 15.23 & 16.43 & 3.06 & 3.03 & 11.32 & 12.10 & 39.53 & 39.39 & 12.66 & 12.68 \\
\hline & & Foliar Zn + B & 15.33 & 16.56 & 3.09 & 3.05 & 11.51 & 12.27 & 39.50 & 39.64 & 12.63 & 12.83 \\
\hline & & ean & 15.18 & 16.40 & 3.05 & 3.03 & 11.25 & 12.03 & 39.53 & 39.56 & 12.70 & 12.71 \\
\hline & an thre & eks & 14.94 & 16.13 & 3.03 & 3.03 & 10.99 & 11.81 & 39.57 & 39.56 & 12.67 & 12.70 \\
\hline
\end{tabular}

Fayoum J. Agric. Res. \& Dev., Vol. 29, No.1, January, 2015 
Emara M.A.A. et al.,

Cont. Table (5):

\begin{tabular}{|c|c|c|c|c|c|c|c|c|c|c|c|c|}
\hline \multicolumn{3}{|c|}{ Characters } & \multicolumn{2}{|c|}{\begin{tabular}{|c|}
$\begin{array}{c}\text { No. of } \\
\text { open } \\
\text { bolls/plant }\end{array}$ \\
\end{tabular}} & \multicolumn{2}{|c|}{$\begin{array}{c}\text { Boll } \\
\text { weight }(\mathrm{g})\end{array}$} & \multicolumn{2}{|c|}{$\begin{array}{c}\text { Seed cotton } \\
\text { yield } \\
\text { (Kentar/fed.) }\end{array}$} & \multicolumn{2}{|c|}{$\mid \begin{array}{c}\text { Lint } \\
\text { percentage } \\
(\%)\end{array}$} & \multicolumn{2}{|c|}{$\begin{array}{c}\text { Seed index } \\
\text { (g) }\end{array}$} \\
\hline \multicolumn{3}{|c|}{ Treatments } & \multirow[b]{2}{*}{2013} & \multirow[b]{2}{*}{2014} & \multirow[b]{2}{*}{2013} & \multirow[b]{2}{*}{2014} & \multirow[b]{2}{*}{2013} & \multirow[b]{2}{*}{2013} & \multirow[b]{2}{*}{2014} & \multirow[b]{2}{*}{2013} & \multirow[b]{2}{*}{2014} & \multirow[b]{2}{*}{2013} \\
\hline $\begin{array}{c}\text { Irrigation } \\
\text { intervals (A) }\end{array}$ & $\begin{array}{c}\text { Levels } \\
\text { of NPK } \\
\text { (B) }\end{array}$ & $\begin{array}{l}\text { Micronutrients } \\
\text { (C) }\end{array}$ & & & & & & & & & & \\
\hline \multirow{15}{*}{ Four weeks } & \multirow{4}{*}{$\begin{array}{c}60 \mathrm{~N}+ \\
22.5 \\
\mathrm{P}_{2} \mathrm{O}_{5}+ \\
24 \mathrm{~K}_{2} \mathrm{O}\end{array}$} & Control & 14.06 & 15.66 & 2.84 & 2.85 & 9.68 & 10.48 & 39.42 & 39.39 & 12.67 & 2.59 \\
\hline & & Foliar Zn & 14.23 & 15.40 & 2.85 & 2.87 & 9.82 & 10.68 & 39.37 & 39.22 & 12.73 & 2.72 \\
\hline & & Foliar B & 14.23 & 15.46 & 2.91 & 2.87 & 9.95 & 10.72 & 39.93 & 39.13 & 12.76 & 12.78 \\
\hline & & Foliar Zn + B & 14.30 & 15.50 & 2.90 & 2.90 & 10.01 & 10.83 & 39.52 & 39.66 & 12.59 & 12.63 \\
\hline & \multicolumn{2}{|r|}{ Mean } & 14.20 & 15.50 & 2.88 & 2.87 & 9.86 & 10.67 & 39.31 & 39.35 & 12.69 & 12.68 \\
\hline & \multirow{4}{*}{$\begin{array}{c}75 \mathrm{~N}+ \\
30 \mathrm{P}_{2} \mathrm{O}_{5} \\
+ \\
36 \mathrm{~K}_{2} \mathrm{O}\end{array}$} & Control & 14.16 & 15.33 & 2.97 & 2.99 & 10.13 & 10.95 & 39.29 & 39.49 & 12.78 & 12.75 \\
\hline & & Foliar Zn & 14.23 & 15.30 & 2.98 & 3.00 & 10.23 & 11.11 & 39.51 & 39.48 & 12.76 & 12.79 \\
\hline & & Foliar B & 14.43 & 15.36 & 2.98 & 3.00 & \begin{tabular}{|l|}
10.37 \\
\end{tabular} & 11.7 & 39.47 & 39.26 & 12.74 & 12.74 \\
\hline & & Foliar Zn + B & 14.63 & 15.46 & 3.01 & 3.02 & 10.61 & 11.31 & 39.46 & 39.62 & 12.80 & 12.80 \\
\hline & \multicolumn{2}{|r|}{ Mean } & 14.36 & 15.36 & 2.99 & 3.00 & 10.33 & 11.13 & 39.43 & 39.46 & 12.77 & 12.77 \\
\hline & \multirow{4}{*}{$\begin{array}{c}90 \mathrm{~N}+ \\
37.5 \\
\mathrm{P}_{2} \mathrm{O}_{5}+ \\
48 \mathrm{~K}_{2} \mathrm{O}\end{array}$} & Control & 14.46 & 15.40 & 2.98 & 3.00 & 10.43 & 11.18 & 39.57 & 39.59 & 12.59 & 12.92 \\
\hline & & Foliar Zn & 14.70 & 15.53 & 3.00 & 3.01 & 10.66 & 11.35 & 39.50 & 39.68 & 12.71 & 2.78 \\
\hline & & Foliar B & 14.80 & 15.63 & 3.00 & 3.01 & 10.72 & 11.43 & 39.49 & 39.64 & 12.79 & 12.80 \\
\hline & & Foliar Zn + B & 14.83 & 15.63 & 3.02 & 3.03 & 10.79 & 11.51 & 39.64 & 39.55 & 12.60 & 12.60 \\
\hline & & Mean & 14.70 & 15.55 & 3.00 & 3.01 & 10.65 & 11.37 & 39.55 & 39.61 & 12.67 & 12.77 \\
\hline \multicolumn{3}{|c|}{ Mean four weeks } & 14.42 & 15.47 & 2.95 & 2.96 & 10.28 & 11.06 & 39.43 & 39.47 & 12.71 & 12.74 \\
\hline \multirow{3}{*}{$\begin{array}{c}\text { Mean levels } \\
\text { of } N P K(B)\end{array}$} & \multicolumn{2}{|c|}{$60 \mathrm{~N}+22.5 \mathrm{P}_{2} \mathrm{O}_{5}+24 \mathrm{~K}_{2} \mathrm{O}$} & 14.59 & 15.84 & 2.96 & 2.96 & 10.46 & 11.31 & 39.50 & 39.43 & 12.66 & 12.67 \\
\hline & $75 N+3$ & $0 \mathrm{P}_{2} \mathrm{O}_{5}+36 \mathrm{~K}_{2} \mathrm{O}$ & 14.92 & 16.02 & 3.04 & 3.06 & 11.01 & 11.84 & 39.49 & 39.51 & 12.74 & 12.76 \\
\hline & $90 \mathrm{~N}+37$ & $.5 \mathrm{P}_{2} \mathrm{O}_{5}+48 \mathrm{~K}_{2} \mathrm{O}$ & 15.33 & 16.38 & 3.08 & 3.08 & 11.45 & 12.23 & 39.53 & 39.59 & 12.67 & 12.72 \\
\hline & $\begin{array}{r}\begin{array}{r}\text { Con } \\
\text { micronut }\end{array} \\
\end{array}$ & $\begin{array}{l}\text { (without } \\
\text { its application) }\end{array}$ & 14 & 15.93 & 3.00 & 3.02 & 10.77 & 11.59 & 39.52 & 39.49 & 12.67 & 12.73 \\
\hline micronu & & oliar Zn & 14.91 & 16.00 & 3.02 & 3.04 & 10.90 & 11.73 & 39.50 & 39.46 & 12.70 & 12.71 \\
\hline & & Foliar B & 15.01 & 16.11 & 3.04 & 3.03 & 11.02 & 11.84 & 39.46 & 39.46 & 12.71 & 12.74 \\
\hline & Fol & $\operatorname{iar} \mathbf{Z n}+\mathbf{B}$ & 15.10 & 16.28 & 3.06 & 3.05 & 11.20 & 12.01 & 39.56 & 39.62 & 12.69 & 12.69 \\
\hline & Irrigatic & intervals (A) & 0.048 & 0.074 & 0.005 & 0.004 & \begin{tabular}{|l|}
0.102 \\
\end{tabular} & 0.004 & N.S & N.S & N.S & N.S \\
\hline & Level & of NPK (B) & 0.032 & 0.079 & 0.004 & 0.003 & 0.043 & 0.012 & N.S & N.S & N.S & N.S \\
\hline & Micro & nutrients (C) & 0.056 & 0.139 & 0.007 & 0.005 & 0.074 & 0.020 & N.S & N.S & N.S & N.S \\
\hline at 0.05 & & A X B & $\mathbf{0 . 0 3 7}$ & 0.092 & 0.005 & 0.003 & 0.049 & 0.013 & N.S & N.S & N.S & N.S \\
\hline & & A X C & N.S & 0.160 & 0.008 & 0.006 & N.S & 0.023 & N.S & N.S & N.S & N.S \\
\hline & & B X C & N.S & 0.064 & 0.008 & 0.006 & N.S & 0.023 & $\mathbf{N} . \mathbf{S}$ & N.S & N.S & N.S \\
\hline & & X B X C & N.S & 0.112 & 0.014 & 0.010 & 0.086 & 0.040 & N.S & N.S & N.S & N.S \\
\hline
\end{tabular}

\section{C-3- Effect of micronutrients treatments:}

The results in Table (5) show that, foliar application with micronutrients treatments had a significant effect on no. of open bolls/plant, boll weight and seed cotton yield/feddan in 2013 and 2014 seasons, but insignificant effect on lint percentage and seed index in both season. The highest values of no. of bolls/plant (15.10 and 16.28), boll weight (3.06 and $3.05 \mathrm{~g}$ ) and seed cotton yield/feddan (10.20 and 12.01 kentar/feddan) were produced from the foliar application mixtures which contain two elements (Zn-EDTA and B-EDTA) at the start and peak of flowering stage, while the lowest values of no. of bolls/plant (14.78 and 15.93), boll weight (3.00 and $3.02 \mathrm{~g}$ ) and seed cotton yield/feddan (10.77 and 11.59 kentar/feddan) were obtained from control (without foliar application)

Fayoum J. Agric. Res. \& Dev., Vol. 29, No.1, January, 2015 
EFFECT OF WATER STRESS AND FOLIAR FEEDING WITH BORON.

treatment, in 2013 and 2014 seasons, respectively. These results are in accordance with those outlined by overall plant growth, fruit retention, seed cotton yield, yield components Saeed (2000), El-Shazly et al., (2005), Sawan et al., (2006 and 2007), Kassem et al., (2009), Ali et al., (2011), Emara (2012) and El-Gabiery, (2014).

\section{C-4- Effect of interaction:}

Results presented in Table (5) indicate that the interaction between irrigating intervals (A) and NPK levels (B) had significant effect on no. of open bolls/plant, boll weight and seed cotton yield/feddan and insignificant effect on lint percentage and seed index in both seasons.

Data in Table (5) indicate that the interaction between irrigation intervals (A) and foliar feeding with micronutrients (C) gave significant effect on boll weight in both seasons and on no. of open bolls/plant and seed cotton yield/feddan in one season only. This interaction gave insignificant effect on lint percentage and seed index in both seasons.

Results presented in Table (5) indicate that the interaction between levels of NPK (B) and foliar application with micronutrients treatments (C) had significant effect on boll weight in both seasons and no. of open bolls/plant and seed cotton yield/feddan in one season only. While, this interaction gave insignificant effect on lint percentage and seed index in both seasons. The superiority of the high concentration of nutrients with regard to seed cotton yield and its components is mainly attributed to the higher no. of open bolls/plant, heavier bolls and higher seed cotton yield/plant which related to the constituents of nutrients mixture ( $\mathrm{Zn}$ and $\mathrm{B}$ ), which lead to: nutrients enriched the cotton plant with appreciable amount of N, P, K, Zn, and B.

Data in Table (5) indicate that the effect of the interaction between (A) and (B) and (C) was significant on boll weight and seed cotton yield/feddan in both seasons and on no. of open bolls/plant in one season only, but was insignificant on lint percentage and seed index in both seasons.

\section{D- Fiber quality traits:}

The results in Table (6) indicate that irrigation intervals, levels of NPKfertilization, foliar application of some micronutrients and their interactions did not exhibit significant effect on fiber properties under study i.e., fiber length parameters (fiber upper half mean length, uniformity index), micronaire reading and fiber strength in 2013 and 2014 seasons.

The positive effect of the interaction on the studied traits may be attributed to:

* The role of NPK in encouraging the photosynthesis and assimilates accumulation. The positive effect of $\mathrm{N}$ on photosynthetic rate and accumulation of carbohydrates ...etc.

* In addition, $\mathrm{N}$ has a role in building up plant organs through the synthesis of protein.

* The role of $\mathrm{P}$ in photosynthesis and respiration, $\mathrm{P}$ plays a major role in energy storage.

Fayoum J. Agric. Res. \& Dev., Vol. 29, No.1, January, 2015 
Emara M.A.A. et al.,

* In addition, $\mathrm{P}$ is required in large quantities in young cells, such as shoots and root tips, where metabolism is high and cell division is rapid. Phosphorus deficiency cause delay in maturity and reduced seed quality.

* The simulative effect due to the role of potassium on enzymes promotion activity and enhancing the translocation of assimilates and protein. Because $\mathrm{K}$ is needed in photosynthesis and the synthesis of protein, plants lacking $\mathrm{K}$ will have slow and stunted growth. Potassium reduces boll shedding (Zeng, 1996).

* Potassium nutrition had pronounced effect on carbohydrates partitioning by affecting either phloem export of photosynthesis (sucrose) or growth rate of sink and/or sources organ (Cakmak et al., 1994).

* The role of macro and micro nutrients under study, which are known to promote photosynthesis and plant development which reflected on enhancing the quality and seed development and consequently the productivity of unit area. Nutrients (in the form of mixture) enriched the cotton plant with appreciable amount of $\mathrm{Zn}$ and $\mathrm{B}$.

\section{CONCLUSION}

The results obtained in this study could lead us to a package of recommendations, which seemed to be useful for increasing the cotton yield production. It could be concluded the irrigation intervals every two weeks with NPK fertilizer level (90 kg N $+37.5 \mathrm{~kg} \mathrm{P}_{2} \mathrm{O}_{5}+48 \mathrm{~kg} \mathrm{~K} 2 \mathrm{O} /$ fed.) and foliar feeding with $\mathrm{Zn}$ and $\mathrm{B}$ mixture (which contain the two elements at one level for each 2 $\mathrm{g} / \mathrm{L}$ water) at the start and peak of flowering stages for obtaining high productivity of new promising cotton genotype (Giza 86 X 10229) under this study.

Fayoum J. Agric. Res. \& Dev., Vol. 29, No.1, January, 2015 
EFFECT OF WATER STRESS AND FOLIAR FEEDING WITH BORON.

Table (6): Cotton fiber parameters as affected by irrigation intervals, NPK levels and micronutrients treatments as well as their interactions during 2013 and 2014 seasons.

\begin{tabular}{|c|c|c|c|c|c|c|c|c|c|c|}
\hline \multirow{2}{*}{\multicolumn{3}{|c|}{ Characters }} & \multicolumn{4}{|c|}{ Fiber length parameters } & \multirow{2}{*}{\multicolumn{2}{|c|}{$\begin{array}{l}\text { Micronaire } \\
\text { reading }\end{array}$}} & \multirow{2}{*}{\multicolumn{2}{|c|}{ Fiber strength }} \\
\hline & & & \multicolumn{2}{|c|}{$\begin{array}{c}\text { Upper half } \\
\text { mean length } \\
(\text { UHML) }\end{array}$} & \multicolumn{2}{|c|}{$\begin{array}{c}\text { Uniformity } \\
\text { index } \\
(\mathrm{UI} \%)\end{array}$} & & & & \\
\hline Tre: & atments & & & & & & & & & \\
\hline \begin{tabular}{|c|} 
Irrigation \\
intervals \\
(A) \\
\end{tabular} & $\begin{array}{c}\text { Levels } \\
\text { of NPK } \\
\text { (B) }\end{array}$ & $\begin{array}{c}\text { Micronutrient } \\
\text { (C) }\end{array}$ & 2013 & 2014 & 2013 & 2014 & 2013 & 2014 & 2013 & 2014 \\
\hline \multirow{15}{*}{$\begin{array}{c}\text { Two } \\
\text { weeks }\end{array}$} & \multirow{4}{*}{$\begin{array}{c}60 \mathrm{~N}+ \\
22.5 \\
\mathrm{P}_{2} \mathrm{O}_{5}+ \\
24 \mathrm{~K}_{2} \mathrm{O}\end{array}$} & Control & 35.00 & 35.50 & 86.50 & 87.05 & 3.40 & 3.35 & 9.90 & 10.20 \\
\hline & & Foliar Zn & 35.85 & 35.60 & 88.35 & 89.40 & 3.90 & 4.05 & 10.60 & 10.20 \\
\hline & & Foliar B & 34.85 & 36.90 & 88.50 & 88.15 & 4.15 & 3.90 & 10.20 & 10.00 \\
\hline & & Foliar Zn + B & 35.10 & 34.50 & 87.85 & 86.15 & 4.40 & 4.40 & 10.10 & 10.15 \\
\hline & \multicolumn{2}{|r|}{ Mean } & 35.20 & 35.38 & 87.80 & 87.69 & 3.96 & 3.93 & 10.20 & 10.14 \\
\hline & \multirow{4}{*}{$\begin{array}{c}75 \mathrm{~N}+ \\
30 \mathrm{P}_{2} \mathrm{O}_{5} \\
+ \\
36 \mathrm{~K}_{2} \mathrm{O} \\
\end{array}$} & Control & 35.15 & 33.50 & 88.50 & 86.75 & 4.00 & 4.15 & 10.00 & 10.00 \\
\hline & & Foliar Zn & 34.35 & 34.90 & 86.45 & 86.35 & 3.65 & 3.85 & 10.00 & 10.10 \\
\hline & & Foliar B & 34.2 .0 & 35.25 & 85.50 & 87.05 & 4.25 & 3.90 & 10.40 & 10.30 \\
\hline & & Foliar Zn + B & 35.00 & 34.70 & 85.75 & 86.90 & 4.05 & 4.15 & 10.30 & 10.20 \\
\hline & \multicolumn{2}{|r|}{ Mean } & 34.68 & 34.59 & 86.55 & 86.76 & 3.99 & 4.01 & 10.18 & 10.15 \\
\hline & \multirow{4}{*}{$\begin{array}{c}90 \mathrm{~N}+ \\
37.5 \\
\mathrm{P}_{2} \mathrm{O}_{5}+ \\
48 \mathrm{~K}_{2} \mathrm{O}\end{array}$} & Control & 35.25 & 35.45 & 87.25 & 87.05 & 4.10 & 3.85 & 10.10 & 10.30 \\
\hline & & Foliar Zn & 35.60 & 34.56 & 88.20 & 88.90 & 3.75 & 3.45 & 10.30 & 10.00 \\
\hline & & Foliar B & 35.45 & 36.20 & 88.50 & 89.60 & 3.85 & 3.95 & 10.20 & 10.00 \\
\hline & & Foliar Zn + B & 34.50 & 34.75 & 87.75 & 87.35 & 3.65 & 4.10 & 10.20 & 10.15 \\
\hline & & 35.20 & 35.24 & 87.93 & 88.23 & 3.84 & 3.84 & 10.20 & 10.11 \\
\hline \multicolumn{3}{|c|}{ Mean two weeks } & 35.03 & 35.07 & 87.43 & 87.56 & 3.93 & 3.93 & 10.19 & 10.13 \\
\hline \multirow{15}{*}{$\begin{array}{l}\text { Three } \\
\text { weeks }\end{array}$} & \multirow{4}{*}{$\begin{array}{c}60 \mathrm{~N}+ \\
22.5 \\
\mathrm{P}_{2} \mathrm{O}_{5}+ \\
24 \mathrm{~K}_{2} \mathrm{O} \\
\end{array}$} & Control & 35.25 & 35.09 & 86.00 & 87.95 & 3.90 & 4.25 & 10.20 & 10.20 \\
\hline & & Foliar Zn & 34.90 & 35.25 & 86.45 & 85.80 & 4.40 & 3.95 & 10.30 & 10.00 \\
\hline & & Foliar B & 34.40 & 36.00 & 85.30 & 88.20 & 3.80 & 3.85 & 10.00 & 10.60 \\
\hline & & Foliar Zn + B & 36.00 & 36.70 & 86.70 & 89.00 & 3.75 & 3.65 & 10.20 & 10.35 \\
\hline & \multicolumn{2}{|r|}{ Mean } & 35.14 & 35.76 & 86.11 & 87.74 & 3.96 & 3.93 & 10.18 & 10.28 \\
\hline & \multirow{4}{*}{$\begin{array}{c}75 \mathrm{~N}+ \\
30 \\
\mathrm{P}_{2} \mathrm{O}_{5}+ \\
36 \mathrm{~K}_{2} \mathrm{O}\end{array}$} & Control & 35.90 & 35.75 & 87.10 & 88.95 & 3.60 & 3.60 & 10.40 & 10.10 \\
\hline & & Foliar Zn & 34.15 & 34.50 & 87.65 & 88.15 & 3.85 & 3.80 & 10.10 & 10.00 \\
\hline & & Foliar B & 34.70 & 35.45 & 86.25 & 87.65 & 3.85 & 3.85 & 10.00 & 10.20 \\
\hline & & Foliar Zn + B & 34.55 & 34.40 & 86.40 & 86.30 & 4.15 & 4.25 & 10.00 & 10.40 \\
\hline & \multicolumn{2}{|r|}{ Mean } & 34.83 & 35.03 & 86.85 & 87.76 & 3.86 & 3.88 & 10.13 & 10.17 \\
\hline & \multirow{4}{*}{$\begin{array}{r}90 \mathrm{~N}+ \\
37.5 \\
\mathrm{P}_{2} \mathrm{O}_{5}+ \\
48 \mathrm{~K}_{2} \mathrm{O}\end{array}$} & Control & 34.15 & 34.70 & 85.55 & 87.55 & 3.60 & 4.05 & 10.10 & 10.30 \\
\hline & & Foliar Zn & 35.40 & 35.10 & 85.90 & 86.20 & 3.85 & 3.95 & 10.70 & 10.20 \\
\hline & & Foliar B & 33.85 & 35.95 & 86.95 & 86.65 & 3.35 & 3.45 & 10.10 & 10.10 \\
\hline & & Foliar Zn + B & 34.95 & 36.35 & 86.70 & 89.55 & 3.95 & 3.90 & 10.00 & 10.60 \\
\hline & \multirow{2}{*}{\multicolumn{2}{|c|}{ Mean three weeks }} & 34.59 & 35.53 & 86.28 & 87.49 & 3.69 & 3.84 & 10.23 & 10.30 \\
\hline & & & 34.85 & 35.44 & 86.41 & 87.66 & 3.84 & 3.88 & 10.18 & 10.25 \\
\hline
\end{tabular}

Fayoum J. Agric. Res. \& Dev., Vol. 29, No.1, January, 2015 
Emara M.A.A. et al.,

Cont. Table (6):

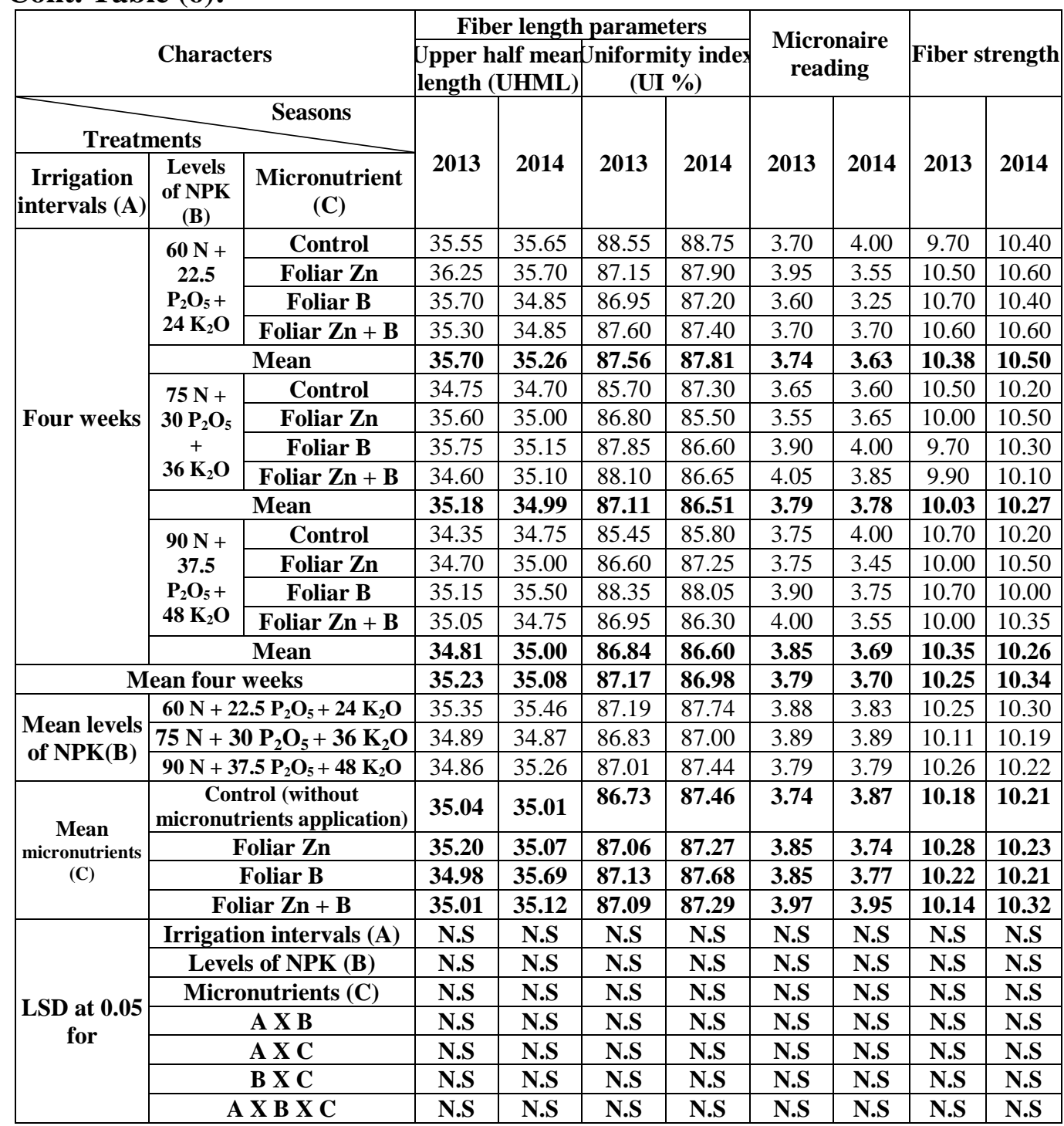

\section{REFERENCES}

A.S.T.M. (2004). American Society for Testing and Materials. Standard Atmosphere for Conditioning and Testing Designation, (D 1776-04).

A.S.T.M. (2012). American Society for Testing and Materials. Designation, (D1447-072012), (D1448-97), (D-1445-67).

Ahmed, A.M. and M.M. Kassem (2008). Cotton response to irrigation intervals under different levels of potassium and nitrogen. Assiut J. of Agric. Sci., 39(5): 171 - 185.

Ali, L.; M. Ali and M. Qamar (2011). Effect of foliar application of zinc and boron on seed cotton yield and economics in cotton-wheat cropping pattern. J. Agric. Res., 49(2): 173 - 180.

Alloway, B.J. (2008). Zinc in soils and crop nutrition. $2^{\text {nd }}$ ed., International Zinc Association, Brussels, Belgium, pp: 135.

Fayoum J. Agric. Res. \& Dev., Vol. 29, No.1, January, 2015 
EFFECT OF WATER STRESS AND FOLIAR FEEDING WITH BORON

Ankerman, D. and L. Large (1974). Soil and Plant Analysis. A \& L. Agriculture Laboratories. Inc., New York. USA.

Baslious, S.I. and K.K. Abdel Malak (1992). Effect of irrigation intervals, nitrogen levels and their interaction on seed cotton yield of Giza 83 and its components. Minia J. Agric. Res. \& Dev., 14(3): 699 - 713.

Blevins, D.G. and K.M. Lukaszewski (1998). Boron in plant structure and function. Annu. Rev. Plant Pysiol. Plant Mol. Biol., 49: 481 - 500.

Cakmak, I. (2000). Possible role of zinc in protecting plant cells from damage by reactive oxygen species. New Phytol., 146(2): 185 - 205.

Cakmak, I.; C. Hengeler and H. Marschner (1994). Partitioning of shoot and root dry weight and carbohydrates in bean plants suffering from phosphorus, potassium and magnesium deficiency. J., Exp. Bot., 45: 1245-1250.

Carvalho, L.H.; N.M. Silva, S.M. Brasil, J.I. Kondo and E.J. Chiavegato (1996). Application of boron to cotton by side dressing and foliar spray. Revista Brasileira de Ciencia do Solo. 20: 265 - 269.

Chapman, H.D. and F.P. Parker (1981). Methods of analysis of soil, plants and water. Univ. California, August, 1981. Second Printing.

Dar, W.D. (2004). Macro-benefits from micronutrients: for grey to green revolution in agriculture. Proc. IFA International Symposium on Micronutrients, 23-25 February, New Delhi, India.

Dong, J.F. (1995). The yield increasing ability of spraying cotton with boron. Henan Nongye Kexue. pp: 3 - 6.

El-Fouly, M.M. (2006). Micronutrients in soils and their roles in plant, animal and human health. Proc. $12^{\text {th }}$ AFA Inter. Fertilizer Forum, 6 - 8 February, Cairo, Egypt.

El-Fouly, M.M. and A.F. Fawzi (1995). Higher and better yields with less environmental pollution in Egypt through balanced fertilizer use. Nutrient Cycling in Agroecosystems, 43(1-3): 1-4.

El-Gabiery, A.E. (2014). Response of cotton plant to non- rooting nutrition. The $1^{\text {st }}$ International Cotton Conference "Challenges to Sustainable Cotton Production \& Guality". Special Issue of Egypt. J. Agric. Res., 92 (1): 233 - 250.

El-Ganaini, S.S.; M.A. Saif-El-Yazal, and S.E. Mohamed (2005). Botanical studies on cotton (Gossypium Vitifolium L.) plants grown under newly reclaimed soils as affected by nitrogen and phosphorus fertilization. Ann. Agric. Sci. Moshtohor. 43(4): 1599 - 1617.

El-Menshawi, M.E. and E.A. El-Sayed (2007). Some trails for increasing cotton yield by foliar application of some micronutrients. J. Agric. Sci. Mansoura Univ., 32(1): 1 - 9 .

EL-Sayed, E.A. (2005). Effect of water stress and potassium fertilizer levels on growth and yield of cotton cultivar Giza 88. J. Agric. Sci., Mansoura Univ., 30(1): 49 - 59.

EL-Shahawy, M.I. and R.R. Abd EL-Malik (1999). Response of Giza 87 cotton cultivar (Gossypium barbadense, L.) to irrigation intervals and nitrogen fertilization levels. Egypt. J. Agric. Res., 77(2): 841 - 856.

El-Shazly, W.M.; R.Kh. Khalifa and O.A. Noval (2005). Response of cotton Giza 89 cultivar to foliar spray with boron, potassium and bioregulator SGA-1. Egypt. J. Appl. Sci., 18(4B): 676 - 699.

Emara, M.A. (2012). Response of cotton growth and productivity to application of potassium and zinc under normal and late sowing dates. J. Plant Prod., Mansoura Univ., 3(3): 509 - 514.

Fayoum J. Agric. Res. \& Dev., Vol. 29, No.1, January, 2015 
Emara M.A.A. et al.,

Freed, R.D. (1991). M Stat-C Microcomputer Statistical Program. Michigan State Univ., East Lansing, Michigan, USA.

Gupta, U.C. (1993). Factors affecting boron uptake by plant. CRC Press, Inc., pp: 87-104.

Halepyati S.R.; A.S. Pujari, B.T. Koppalakar and K.N. Rao (2012). Effect of macro and soluble micronutrients on yield, uptake of nutrients, quality and economics of Bt cotton (Gossypium hirsutum L.,) under irrigation. Karnataka J. Agric. Sci., 25(4): $418-422$.

Hamed, F.S. (2007). Response of cotton cultivar Giza 90 to water stress and NPK levels. Minia J. of Agric. Res. \& Develop., 27(5): 941 - 952.

Hamissa, M.R. and M.E. Abdel-Salam (1999). Fertilizer management for cotton in Egypt. Adv. Agric. Res., Egypt. 2(1): 53 - 63.

Hamoda, S.A.; M.A. Ibrahim and M.A. Emara (2014). Effect of irrigation intervals and NPK fertilizers on growth, yield and quality for new hybrid cotton [Giza 83 (Giza 75 x 5844)] x Giza 80. The $1^{\text {st }}$ International Cotton Conference "Challenges to Sustainable Cotton Production \& Guality". Special Issue of Egypt. J. Agric. Res., 92 (1): 111 - 123.

Hamoda, S.A.; M.A. Ibrahim and M.A. Emara. (2013). Impact of water stress and planting patterns on cotton grown in Middel Egypt. Special Issue of J. of the Adv. in Agric. Res., (1): $1-11$. The $2^{\text {nd }}$ Alexandria International Cotton Conference, Faculty of Agric., Saba Basha, Alexandria Univ., Alex. 17 - 18 April, 2012.

Hearn, A.B. (1981). Cotton nutrition. Field Crop Abst., 34(1): 11 - 34.

Heitholt, J.J. (1994). Effect of foliar urea- and triazone- nitrogen, with and without boron, on cotton. J. Plant. Nutr., 17: 57 - 70.

Howard, D.D.; C.O. Gwathmey and C.E. Sams (1998). Foliar feeding of cotton: Evaluating potassium sources, potassium solution buffering and boron. Agro. J., 90: 740 - 766.

Kassem, M.A.; M.A. Emara and S.A. Hamoda. (2009). Growth and productivity of Giza 80 cotton cultivar as affected by foliar feeding with boron and zinc. J. Agric. Sci., Mansoura Univ., 34(2): 967 - 975.

Lale, E. and Y. Emine. (2011). The effect of Zinc application methods on seed cotton yield, lint and seed quality of cotton (Gossypium hirsutum L.,) in east Mediterranean region of Turkey. African J. of Biotechnology. 15 August, 10(44): 8782 - 8789

Malakouti, M.J. (2006). Effect of micronutrients in ensuring efficient use of macronutrients. Proc. IFA Agriculture Conf., Optimizing resource use efficiency for sustainable intensification of agriculture, 27 February to 2 March, Kunming, China.

Niaz, A.; M. Ibrahim, N. Ahmed and A. Anwar (2002). Boron contents of light and medium texture soils and cotton plants. Inter. J. Agric. \& Biol., 4(4): 534 - 536.

Oosterhuis, D.M. and L.A. Venter (1976). Cotton boron trail. Ministry of Agriculture, Salisbury, Rhodesia. Cotton Research Institute Annual Report. pp: 1416.

Policepatil, A.; B. Chittapur, N. Manjunatha and V. Paramesh (2009). Response of Bt cotton hybrids for targeted yield under Northern transitional zone of Karnataka. J. of Crop and Weed. 5(1): 313 - 315.

Rengel, Z. (2007). Role of zinc in plant physiology. Proc. Zinc International Conf. Improving crop production and human health, 24 - 25 May. Istanbul, Turkey.

Fayoum J. Agric. Res. \& Dev., Vol. 29, No.1, January, 2015 
EFFECT OF WATER STRESS AND FOLIAR FEEDING WITH BORON

Roberts, R.K.; J.M. Gersman and D.D. Howard (2000). Soil and foliar applied boron in cotton production: An economic analysis. J. Cotton Sci. 4: 171 - 177.

Rosolem, C. and A. Costa (1999). Boron nutrition and growth of cotton as a function of temporary boron deficiency. Anain II Congresso Brasileiro de Algodao: Oalgodao no seculo XX, perspectivas para a seculo XXI, Ribeirao Preto, SP, Brasil, 5 - 10 Setembro, 403 - 406.

Saeed, M.A. (2000). Physiological response of cotton cultivar Giza 80 to foliar spray of boron. Minufiya J. Agric. Res., 25(5): 1185 - 1197.

Sawan, Z.M.; M.H. Mahmoud and Amal H. El-Guibali (2006). Response of yield, yield components, and fiber properties of Egyptian cotton (Gossypium barbadense, L.) to nitrogen fertilization and foliar applied potassium and mepiquat chloride. J. of Cotton Sci., 10: 224 - 234.

Sawan, Z.M.; S.A. Hafez, A.E. Basyony and A.E. Alkassas (2007). Cottonseed: protein, oil yields, and oil properties as influenced by potassium fertilization and foliar application of zinc and phosphorus. Grasas Y Aceites, 58 (1), ENERO-MARZO, 40 - 48, ISSN: 0017 - 3495.

Seadh, S. E.; M. H. El-Hendi; H. A. Abd El-Aal and Shaimaa O. S. El -Sayed (2012).Effect of NPK rates and humic acid applications on growth of Egyotian cotton. J. Plant production, Mansoura Univ., Vol. 3 (8):2287-2299.

Sema, B.; T. Bahar, E. Remzi, Y. Mustafa and B. Yüksel (2012). The determination effects of potassium and zinc application to rate of photosynthesis, fiber yield and quality on cotton. $11^{\text {th }}$ Meeting of inter-regional cooperative research network on cotton for the Mediterranean and Middle East Regions. November 5 - 7, Antalya, pp: 25.

Snedecor, G.W. and W.G. Cochran (1988). Statistical Methods. $7^{\text {th }}$ Ed. Press, Iowa, Ames, U.S.A. pp: 225 - 269.

Sun, Z.Y. and C.J. Xu (1986). Soil available B in the south of Hebei province and application of boron to cotton. J. Soil Sci., 17: 130 - 132.

Suresh, S. and S. Kumar (2005). Magnesium and zinc effects on yield and nutrients uptake by cotton. Adv. Pl. Sci., 18 (1): 249 - 252.

Tomar, R.; A. Kushwaha, R. Julka and K. Mandloi (2000). Productivity of upland cotton (Gossypium hirsutum, L) genotypes under different levels of fertility and spacing. Indian J. Agron., 45(4): 776 - 781.

Uchida, R. (2000). Essential nutrients for plant growth: Nutrient functions and deficiency symptoms. Plant Nutrient Management in Hawaiis Soils. Approaches for tropical and Subtropical Agriculture. Chapter 3: 31 - 55.

Wang, H. and Y. Jin (2005). Photosynthetic rate, chlorophyll fluorescence parameters and lipid peroxidation of maize leaves as affected by zinc deficiency. Photosynthetica, 43: 591 - 596.

Wazir, M.Y. and A.M. Shahbaz (2013). Role of foliar feeding of micronutrients in yield maximization of cotton in Punjab. Turkish J. Agric. and Forestry. 37(4): 420 - 426.

Zeng, Q. (1996). Experimental study on the efficiency of $\mathrm{K}$ fertilizer applied to cotton in areas with cinnamon soil or aquic soil. China Cottons, 23: 12.

Zhao, D. and M. Oosterhuis (2003). Cotton growth and physiological response to boron deficiency. J. Plant Nutrition, 26(4): 855 - 867.

Fayoum J. Agric. Res. \& Dev., Vol. 29, No.1, January, 2015 
Emara M.A.A. et al.,

تأثير الإجهاد المائي والرش بيعض العناصر الصغرى تحت مستويات مختلفة من التسميا علي نمو ومحصول القطن

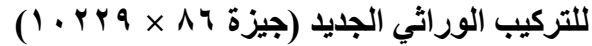

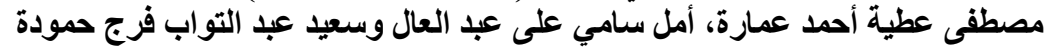

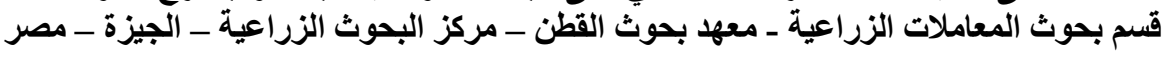

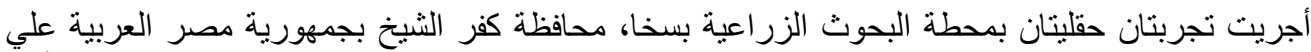

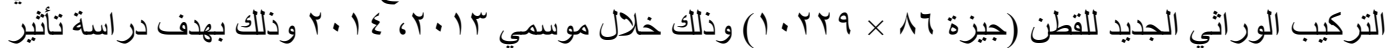

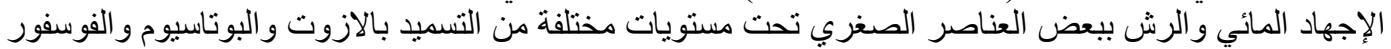

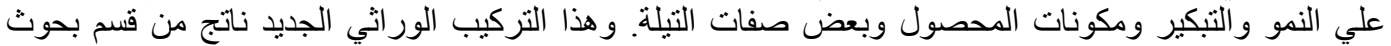
تربية القطن وفى مرحلة اعداد التوصيات الفنيه لـ لهـ.

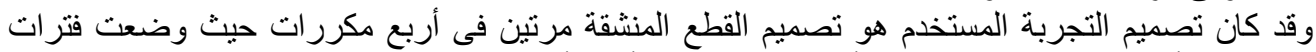

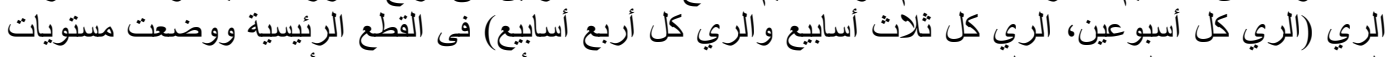

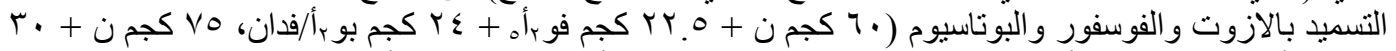

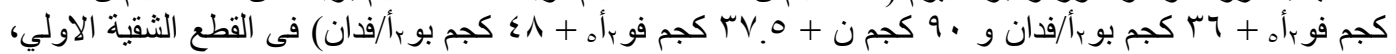

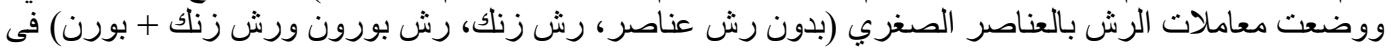

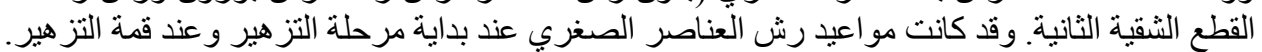

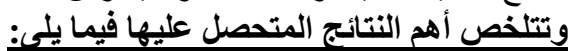

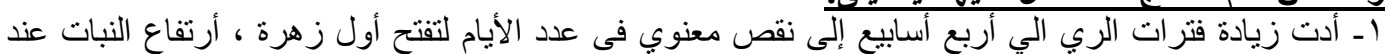

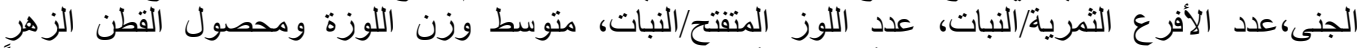

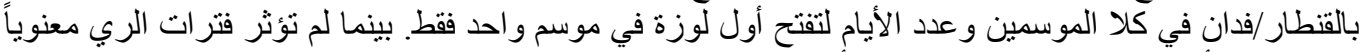

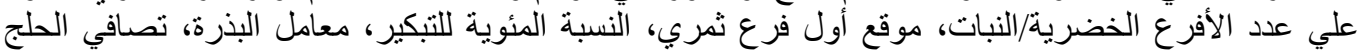

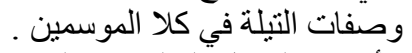

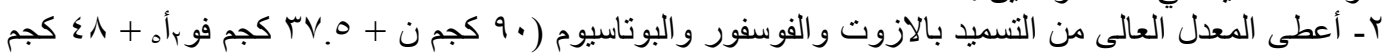

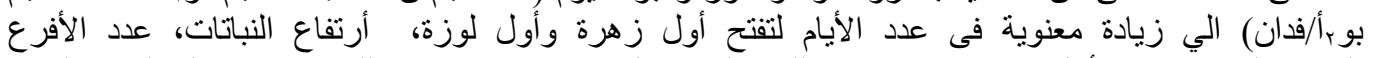

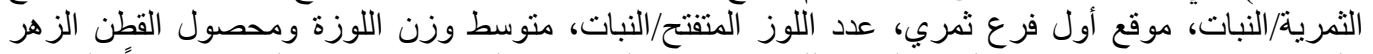

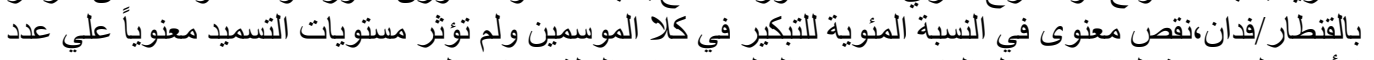

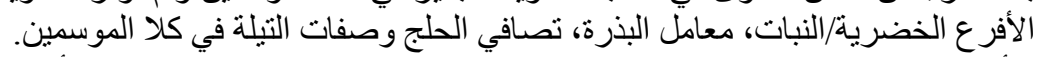

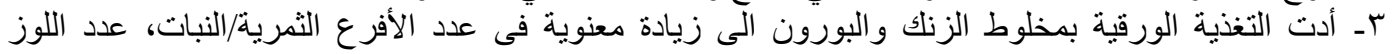

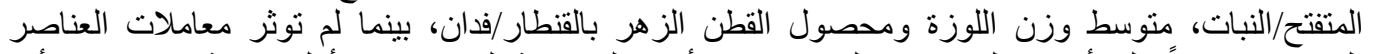

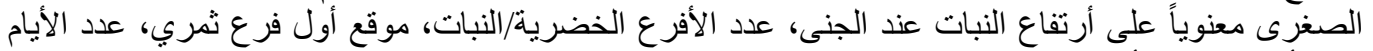

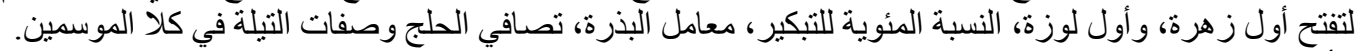

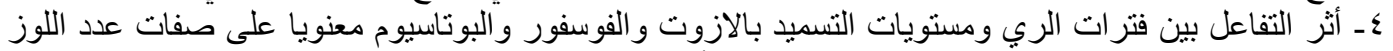

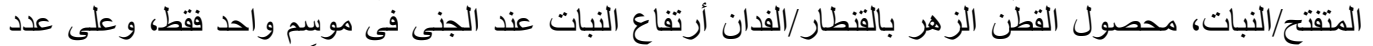

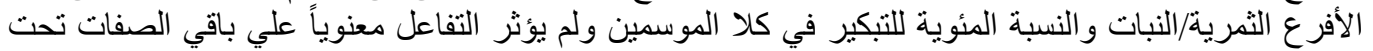

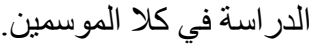

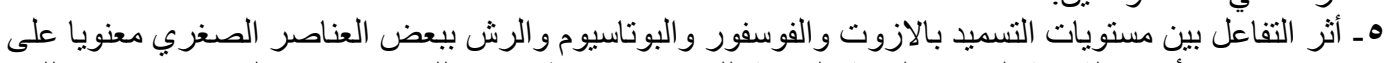

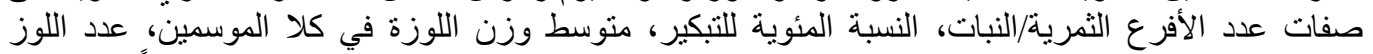

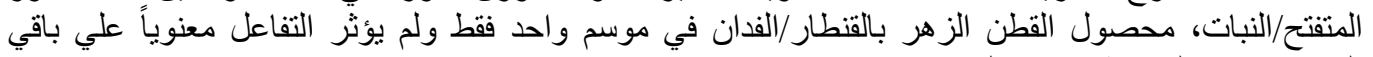
الصفات تحت الدر اسة في كلا الموسمين.

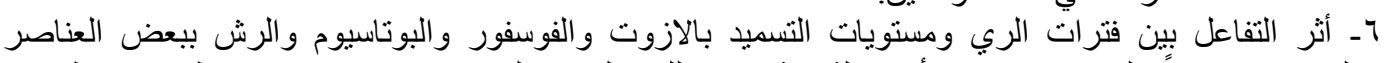

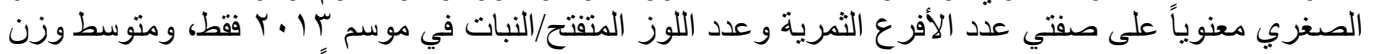

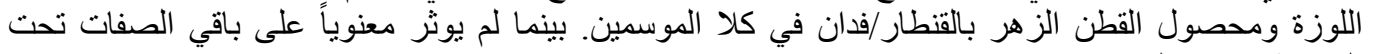

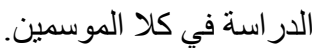
التوصية:

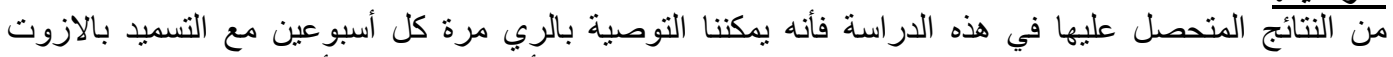

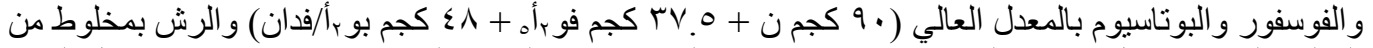

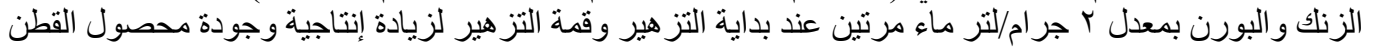

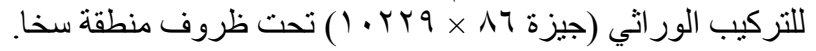

Fayoum J. Agric. Res. \& Dev., Vol. 29, No.1, January, 2015 